\title{
Large-scale fluctuations of PSBL magnetic flux tubes induced by the field-aligned motion of highly accelerated ions
}

\author{
E. E. Grigorenko ${ }^{1}$, T. M. Burinskaya ${ }^{1}$, M. Shevelev ${ }^{1}$, J.-A. Sauvaud ${ }^{2}$, and L. M. Zelenyi ${ }^{1}$ \\ ${ }^{1}$ Space Research Institute of RAS, Profsoyuznaya str. 84/32, Moscow, 117997, Russia \\ ${ }^{2}$ Centre d'Etudie Spatial des Rayonnements, BP 4346, 9 avenue du colonel Roche 310289 Toulouse cedex 4, France
}

Received: 26 February 2010 - Revised: 1 June 2010 - Accepted: 4 June 2010 - Published: 11 June 2010

\begin{abstract}
We present a comprehensive analysis of magnetic field and plasma data measured in the course of 170 crossings of the lobeward edge of Plasma Sheet Boundary Layer (PSBL) in the Earth's magnetotail by Cluster spacecraft. We found that large-scale fluctuations of the magnetic flux tubes have been registered during intervals of propagation of high velocity field-aligned ions. The observed kink-like oscillations propagate earthward along the main magnetic field with phase velocities of the order of local Alfvén velocity and have typical wavelengths $\sim 5-20 R_{\mathrm{E}}$, and frequencies of the order of $0.004-0.02 \mathrm{~Hz}$. The oscillations of PSBL magnetic flux tubes are manifested also in a sudden increase of drift velocity of cold lobe ions streaming tailward. Since in the majority of PSBL crossings in our data set, the densities of currents corresponding to electron-ion relative drift have been low, the investigation of Kelvin-Helmholtz (K-H) instability in a bounded flow sandwiched between the plasma sheet and the lobe has been performed to analyze its relevance to generation of the observed ultra-low frequency oscillations with wavelengths much larger than the flow width. The calculations have shown that, when plasma conditions are favorable for the excitation of K-H instability at least at one of the flow boundaries, kink-like ultra-low frequency waves, resembling the experimentally observed ones, could become unstable and efficiently develop in the system.
\end{abstract}

Keywords. Magnetospheric physics (Magnetotail boundary layers)

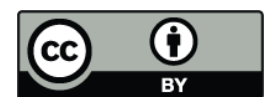

Correspondence to: E. E. Grigorenko (elenagrigorenko2003@yahoo.com)

\section{Introduction}

The Plasma Sheet Boundary Layer (PSBL) represents an interface region separating hot and mostly isotropic plasma of the Central Plasma Sheet (CPS) and the lobe region, which is void of hot plasma but may contain cold ion beams slowly moving tailward (e.g. Sharp et al., 1981). Usually the PSBL is a very dynamic region, where field-aligned ion beams and flows streaming with high parallel velocities $(\geq 1000 \mathrm{~km} / \mathrm{s})$ are often registered (e.g. Lui et al., 1977; DeCoster and Frank, 1979; Andrews et al., 1981; Williams, 1981; Eastman et al., 1984; Baumjohann et al., 1988; Parks et al., 1998; Grigorenko et al., 2002). Recent multipoint observations performed by Cluster spacecraft in the Earth's magnetotail have demonstrated that high-energy ions propagating in the PSBL represent spatial rather than temporal structures, at least during the quiet or moderately disturbed geomagnetic periods. In such cases, the short time of their observations may be caused not only by PSBL flappings in the southnorth direction but also by oscillations of the magnetic tubes in the dawn-dusk direction. It was shown that such oscillations were caused by a magnetic perturbations propagating along the magnetic field lines with local Alfvén velocities (e.g. Grigorenko et al., 2007).

The presence of Alfvén waves in the different regions of the magnetotail was reported in many papers. For example, the events of the large Poynting flux carried by Alfvén waves in the near-Earth PSBL at geocentric distances $4-6 R_{\mathrm{E}}$ were reported by Wygant et al. (2000) and by Keiling et al. (2000, 2001) for active geomagnetic intervals. The periods of these waves were estimated as $\sim 20-60 \mathrm{~s}$. It was also shown that the electromagnetic energy transported by the Alfvén wave down to the polar cap was comparable to the one of an electron aurora. These observations demonstrated the importance of Alfvén waves as a carrier of energy from the magnetotail to the ionosphere, at least during substorm periods. Alfvén

Published by Copernicus Publications on behalf of the European Geosciences Union. 

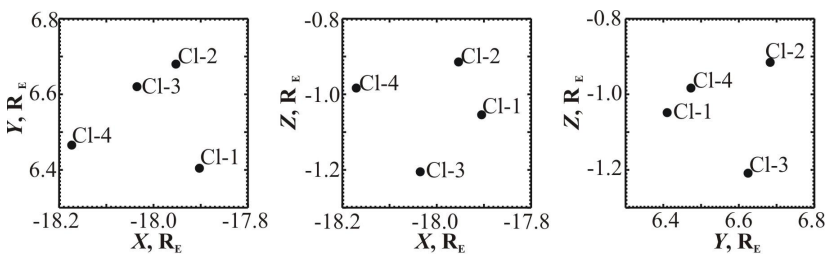

Fig. 1. Cluster position in the GSE coordinate system between 08:15-08:30 UT on 22 September 2001.

waves associated with the bursty bulk flows were studied by Angelopoulos et al. (2002). Takada et al. (2005) reported the observation of Alfvén waves in frequency range $0.01-0.1 \mathrm{~Hz}$ propagating inside the PSBL, the Plasma Sheet (PS) and the CPS. These waves were associated with the hot ion beams having a significant temperature anisotropy, $T_{\mathrm{par}} / T_{\mathrm{per}}<1$. The authors suggested that these waves were generated by ion cyclotron instability. Sauvaud et al. (2004) observed the large Alfvén waves propagating in the PSBL in association with a high speed field-aligned PSBL ion beam. These waves had periods $\sim 80-90$ s and their wavelengths were of the order of $7 R_{\mathrm{E}}$. In the present paper we report the observations of the Alfvén waves propagating earthward in the lobe-PSBL interface with significantly lower frequencies, than the ones studied in the papers mentioned above. We show that these waves are associated with high velocity field-aligned plasma flows streaming earthward along the lobeward edge of PSBL, i.e. along the separatrix magnetic flux tube separating the lobe magnetic field lines devoid of energetic ions and PSBL flux tubes containing high-energy ions. It is necessary to note that high-energy ions propagating at the lobeward edge of the PSBL have velocity distribution functions strongly collimated in pitch-angles. Thus, their energy is mostly concentrated in the form of the kinetic energy of their field-aligned motion.

The presence of PSBL perturbations can be identified due to the increase of energy of cold lobe ions, which are usually not detectable, because of their very low energies. However, in the presence of perturbations of PSBL magnetic tubes associated with Alfvén waves, this cold ion population may acquire a sufficiently large drift velocity and its energy may increase up to a few keV (Mukai et al., 1994; Sauvaud et al., 2004; Zelenyi et al., 2004; Keiling et al., 2006; Grigorenko et al., 2007).

In this paper, we present case and statistical studies of the magnetic perturbations registered in the PSBL during intervals of propagation of high-energy field-aligned ions in the PSBL. It was revealed that the sudden increases of drift velocity of cold lobe ions observed near the lobeward edge of the PSBL were always associated with the propagation of high velocity field-aligned plasma flows and kink-like magnetic fluctuations propagating earthward with the practically local Alfvén velocity. To perform this analysis we used three-point ion measurements by Cluster ion spectrometers
(CIS, Reme et al., 2001), including measurements of threedimensional ion velocity distribution functions and moments collected every $12 \mathrm{~s}$ by the hot ion analyzer (HIA) onboard Cluster-1 and -3 (further mentioned as $\mathrm{Cl}-1$ and $\mathrm{Cl}-3$ ), and the $\mathrm{H}^{+}$and $\mathrm{O}^{+}$moments compiled for $8 \mathrm{~s}$ by the composition and distribution function analyzer (CODIF) onboard Cl-1 and Cl-4. The magnetic field data were obtained with $4 \mathrm{~s}$ resolution by the fluxgate magnetometer (FGM) (Balogh et al., 2001).

Taking into account the results of our analysis of experimental data, we conclude that a development of KelvinHelmholtz $(\mathrm{K}-\mathrm{H})$ instability is the most likely mechanism for the generation of the observed ultra-low frequency kink-like oscillations. On the basis of a model of three (the lobe, the PSBL, the PS) uniform compressible plasma regions, the effects of different plasma parameters in these regions on the development of the K-H instability are discussed.

\section{Alfvén-type perturbations of the PSBL magnetic flux tubes associated with propagation of high velocity field-aligned ions}

On 22 September 2001 between 08:15-08:30 UT Cluster was located in the Southern Hemisphere at $[18,6.5,-1] R_{\mathrm{E}}$ in Geocentric Solar Ecliptic (GSE) coordinate system (Fig. 1) and between 08:20-08:25 UT it experienced a short encounter with the PSBL. Unless noted we use GSE for the following plots. Figure 2 shows Energy-Time (E-T) ion spectrogram measured by the HIA instrument onboard Cluster1, E-T spectrograms of hydrogen and oxygen ions obtained by the CODIF mass-spectrometer onboard Cluster- 4 and E$\mathrm{T}$ ion spectrogram measured by the HIA spectrometer onboard Cluster-3. Cluster-1 and Cluster-4, being located closer to the PS than Cluster-3, crossed the lobeward edge of the PSBL and observed two ion populations with energies below $2 \mathrm{keV}$ and more than $10 \mathrm{keV}$. The high-energy population consists of field-aligned ions streaming earthward with $V \|>2000 \mathrm{~km} / \mathrm{s}$ (see 2-D cuts of ion velocity distribution functions in $\left(V_{\perp}, V \|\right)$ plane measured by the HIA spectrometer onboard Cluster-1 and presented in the right part of Fig. 2). The low-energy ion population consists mainly of $\mathrm{H}^{+}$and $\mathrm{O}^{+}$ions moving slowly tailward with $V X \sim-50 \mathrm{~km} / \mathrm{s}$. At the moment of Cluster exit from the PSBL to the lobe, the energy of the cold ions increased. This phenomenon is more clearly seen for oxygen ions, whose energy values reach their maximum $(\sim 2000 \mathrm{eV})$ just at the moment when Cluster-1, -4 cross the lobeward edge of the PSBL ( at 08:22:50 UT). Although Cluster-3 did not cross the PSBL and did not observe the high velocity ions it, nevertheless, observed the increase of energy of cold ions simultaneously with Cluster- $1,-4$.

Figure 3 displays from top to bottom: the density of highenergy ions $(>5 \mathrm{keV}) ; V X$ component and three components of perpendicular velocities calculated for low-energy 


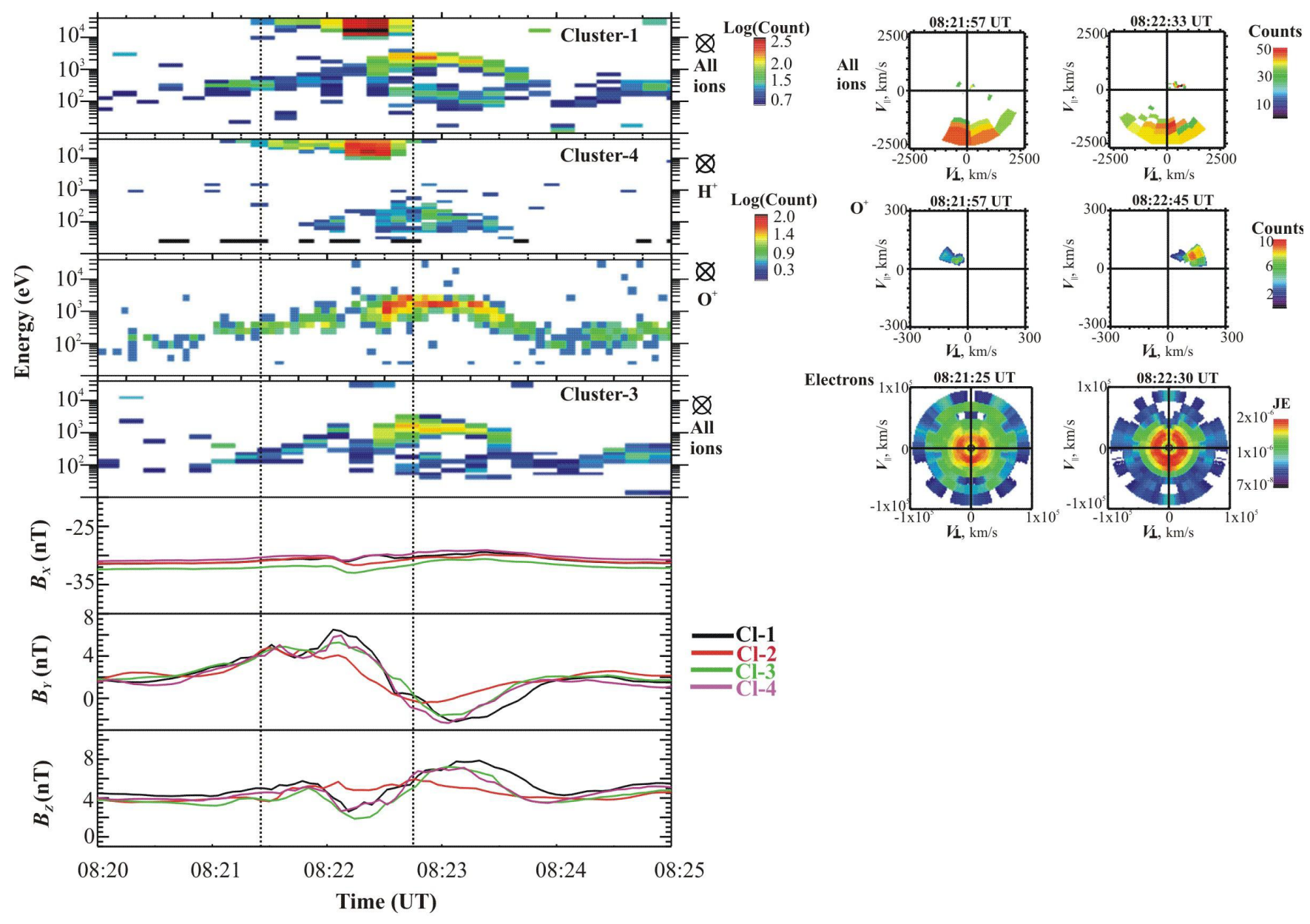

Fig. 2. Left part of the figure (from top to bottom): Energy-Time (E-T) spectrograms of ions (all species), protons and oxygen ions registered by three Cluster s/c; three components of the magnetic field measured by four Cluster s/c. An interval of short PSBL excursion is marked by dashed lines. Right part of the figure (from top to bottom): 2-D cuts of velocity distribution functions of ions (HIA data from Cluster-1) and of oxygen ions (CODIF data from Cluster-4) in $\left(V \|, V_{\perp}\right)$ plane; pitch-angle distribution of electrons (PEACE data from Cluster-2) measured within the interval of interest.

$(<2 \mathrm{keV})$ and high-energy ion components; Y-component of the electric field, deduced from the plasma velocity and magnetic field data, and the dawn-dusk component of electric field, measured by the Cluster Electric Field and Wave experiment (EFW) (e.g. Gustafsson et al., 2001) from Cluster-1. As evidenced by Fig. 3 and by 2-D velocity distribution functions of oxygen ions, presented in Fig. 2, the observed energy enhancement of cold ions is caused by the increase of their velocity perpendicular to the background magnetic field. Actually, during the entire time interval $V X$, component of the cold ion velocity almost does not change, while the absolute value of the perpendicular velocity $V_{\perp} \approx \sqrt{V_{Y}^{2}+V_{Z}^{2}}$ increases up to $155 \mathrm{~km} / \mathrm{s}$, which corresponds to the observed energy enhancement of oxygen ions up to $2 \mathrm{keV}$ (see Fig. 3be). Below we demonstrate that the presence of high velocity field-aligned ions in the lobe-PSBL interface and the increase of energy of cold lobe ion population are related phenomena.
During the interval of high-energy ion observation (in Fig. 3 it is shaded in grey) $V X$ components of high-energy and low-energy ion populations were drastically different (up to 50 times). However, their perpendicular velocities were similar and experienced similar variations. With a purpose to verify this, we calculated a coefficient of correlations $k_{m}$ between the perpendicular velocities of low-energy $\left(V_{\perp \text { low }}\right)$ and high-energy $\left(V_{\perp \text { high }}\right)$ ions as:

$k_{m}=\frac{\sum_{i}\left(V_{\perp \text { low }}^{(m, i)}-<V_{\perp \text { low }}^{(m)}>\right) \cdot\left(V_{\perp \text { high }}^{(m, i)}-<V_{\perp \text { high }}^{(m)}>\right)}{\sqrt{\sum_{i}\left(V_{\perp \text { low }}^{(m, i)}-<V_{\perp \text { low }}^{(m)}>\right)^{2}} \cdot \sqrt{\sum_{i}\left(V_{\perp \text { high }}^{(m, i)}-<V_{\perp \text { high }}^{(m)}>\right)^{2}}},(1)$

where index $m$ corresponds to $X, Y$, or $Z$ GSE component of ion perpendicular velocity, index $i$ denotes a particular time moment of the time series under study, $\left\langle V_{\perp \text { low }}\right\rangle$ and $<V_{\perp \text { high }}>$ are values of the $m$-component of perpendicular velocity of low- and high-energy ions averaged for the 


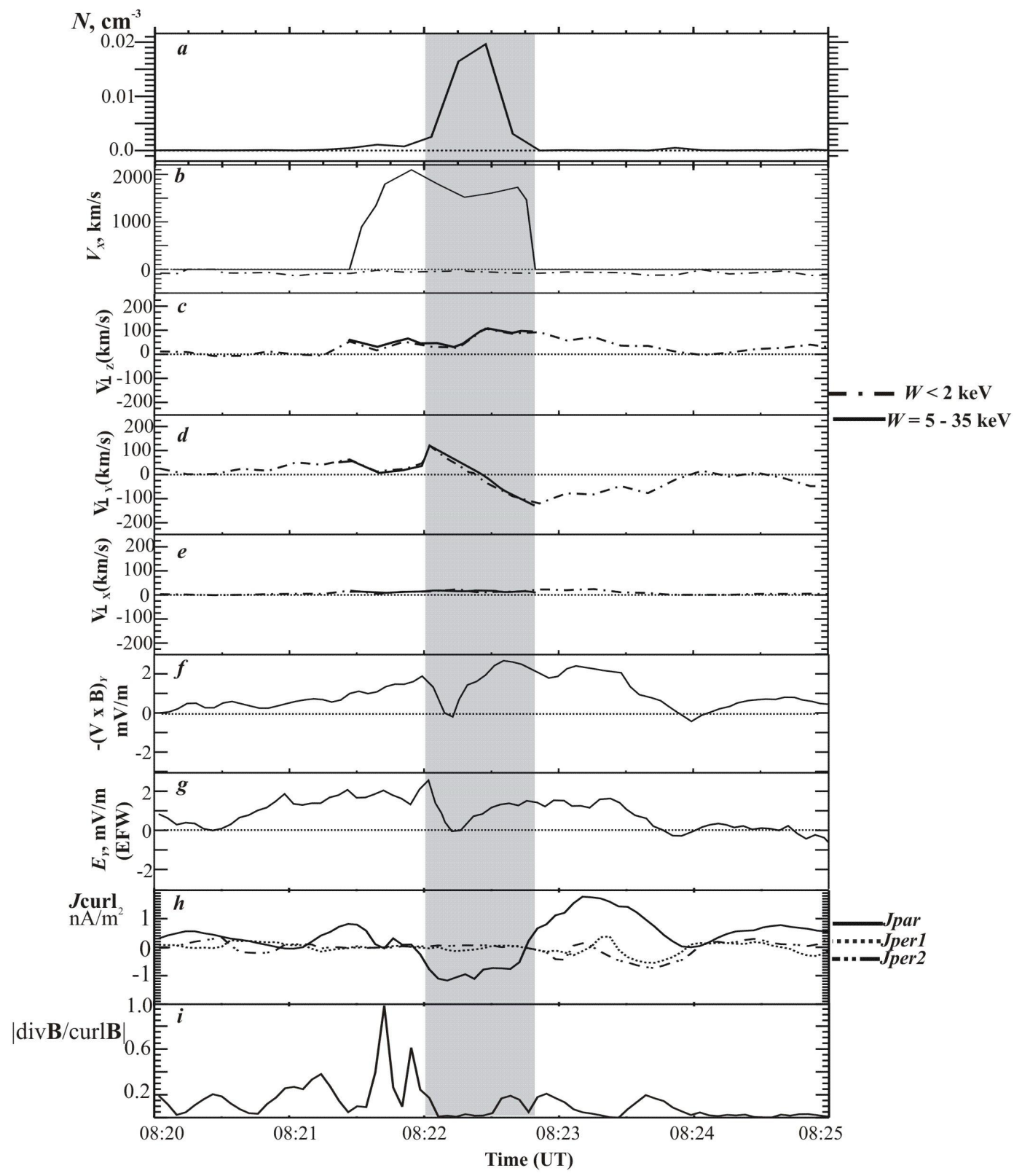

Fig. 3. From top to bottom: (a) Density, $N$, of high-energy ions $(W>5 \mathrm{keV})$; (b-e) the X-component of velocity and three components of perpendicular velocities of low-energy ions (30-2000 eV, shown by dash-dot line) and high-energy ions ( $>5 \mathrm{keV}$, displayed by solid line); (f) the $Y$ GSE-component of the electric field calculated from the magnetic field and velocity of oxygen ions; (g) the dawn-dusk component of the electric field measured by the EFW instrument; (h) three components of the electric current calculated by the curlometer technique (parallel Jpar and perpendicular Jper1, Jper2 to the magnetic field); (i) values of $|\operatorname{div} \boldsymbol{B} / \operatorname{curl} \boldsymbol{B}|$. The interval of interest is shaded in grey.

interval of interest. The coefficients of correlation were calculated for the interval 08:21:25-08:22:40 UT. We obtained the following values of correlation coefficients: $k X=0.85$; $k Y=0.92$ and $k Z=0.73$. The correspondence between variations of perpendicular velocities of high- and low-energy ions indicates the common physical process that is responsible for the perturbations of the magnetic tubes populated by both ion populations. A similar phenomenon was observed by Sauvaud et al. (2004) and it was suggested that the dynamics of low-energy ions was controlled by the motion of the high-energy ion component. A perpendicular motion of the magnetic flux tubes induces the electric field $\boldsymbol{E}=\boldsymbol{V} \times \boldsymbol{B}$ that forces low-energy ions to drift with $\boldsymbol{V}=\boldsymbol{E} \times \boldsymbol{B} / B^{2}$. Note, that the dawn-dusk component of the electric field deduced from the oxygen velocity and magnetic field data, $E Y=-(\boldsymbol{V} \times \boldsymbol{B})_{Y}$ and the $Y$-GSE component of the electric 

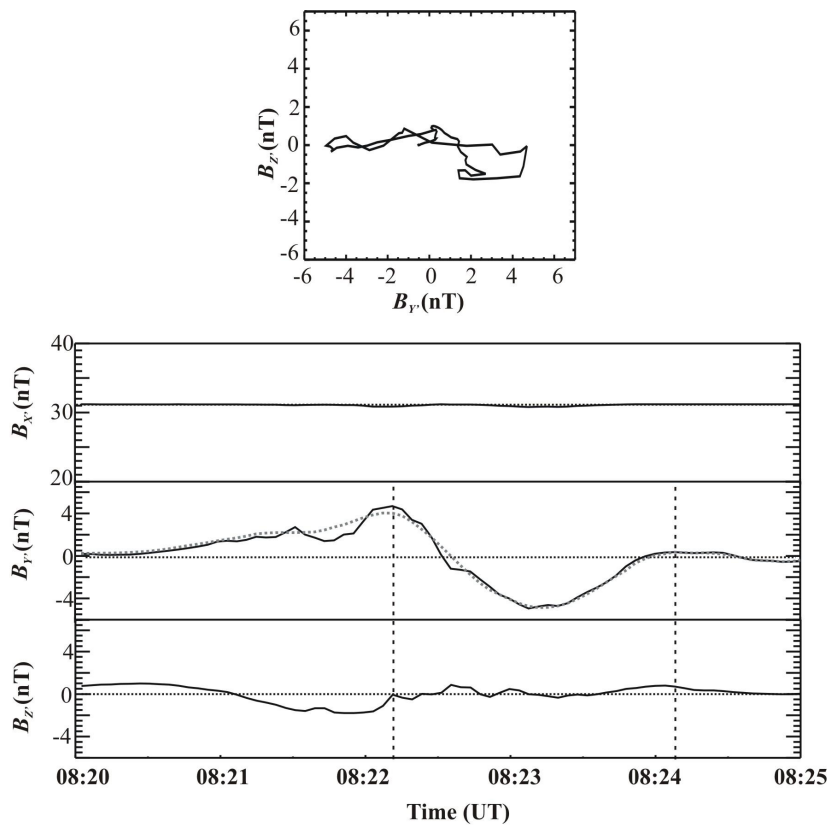

Fig. 4. Hodogram of the magnetic field (Cluster-1 data) in a plane perpendicular to the mean magnetic field direction, $B X^{\prime}$, and three components of $\boldsymbol{B}$ in a new coordinate system $X^{\prime} Y^{\prime} Z^{\prime}$. Grey dotted line displays $B Y^{\prime}$ profile smoothed by the sliding average technique. Vertical dashed lines mark a period of the magnetic perturbation.

field measured by the EFW instrument although not identical, are in quite good agreement (see Fig. 3f, g), and enhancements of the electric field more or less coincide with the increases of ion drift velocity.

Figure 3i shows the current density of field-aligned and of two perpendicular components of the electric current $\left(J_{\text {curl }}\right)$ obtained from the estimation of $(\nabla \times \boldsymbol{B})$ using the reciprocal vectors of the Cluster tetrahedron (e.g. Chanteur, 1998). For the interval of high-energy ion observation a ratio between $(\nabla \cdot \boldsymbol{B})$ and $(\nabla \times \boldsymbol{B})$ was less than 0.2. During this interval the field-aligned current carried by high-energy ions is negative, i.e. is directed earthward and its current density reaches $5.5 \mathrm{nA} / \mathrm{m}^{2}$ (not shown). However, at the same time, the absolute value of density of field-aligned current, estimated as $(\nabla \times \boldsymbol{B})$, does not exceed $1.5 \mathrm{nA} / \mathrm{m}^{2}$. This may imply that during the interval of high-energy ion observation the relative drift velocity of field-aligned ions and electrons was small. Indeed, according to the moments of the electron 3-D phase space distributions obtained by Plasma Electron and Current Experiment (PEACE) (Owen et al., 2001) onboard Cluster-4 for this interval, the electron parallel velocity was directed earthward (as well as the ion one) and it was about $1500 \mathrm{~km} / \mathrm{s}$ (not shown). The small values of electron bulk velocity are due to the absence of energetic uni-directional electron beams for this event. Actually, electron pitch-angle distribution functions measured within the interval of inter-
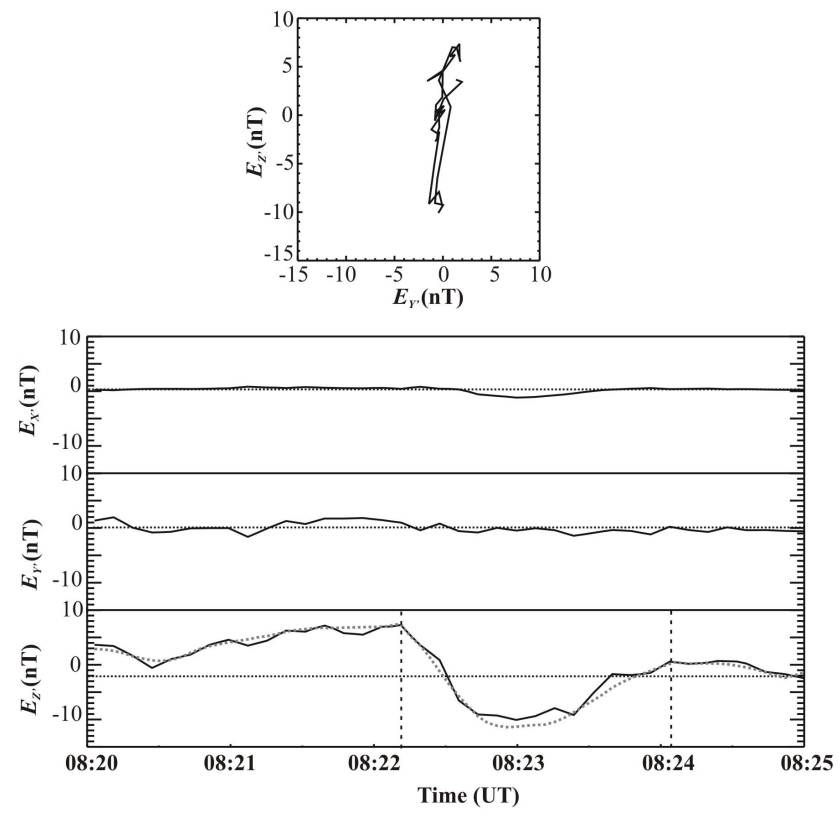

Fig. 5. Hodogram of the electric field $\boldsymbol{E}=-(\boldsymbol{V} \times \boldsymbol{B})$ deduced from the oxygen velocity (CODIF data from Cluster-1) in a plane perpendicular to the main component $E X^{\prime}$ and three components of $\boldsymbol{E}$ in the new coordinate system $X^{\prime} Y^{\prime} Z^{\prime}$. Grey dotted line displays $E Z^{\prime}$ profile smoothed by the sliding average technique. Vertical dashed lines mark a period of the perturbation.

est by Cluster-2 (see Fig. 2) are more or less symmetrical along the magnetic field. Thus, in this event, the high-energy PSBL population may be considered as a high velocity fieldaligned plasma flow, rather than a high velocity ion beam. In the next section we compare statistically the values of total field-aligned currents estimated for each particular event from our data set as $(\nabla \times \boldsymbol{B}) \|$ and the values of field-aligned currents produced by PSBL ions (calculated from the density and parallel velocity of high - energy ions), and show that in the majority of cases under study high velocity field-aligned plasma flows, rather than beams, were observed in the PSBL.

Figures 4 and 5 display the hodographs and variations of the magnetic and electric field, deduced from the oxygen velocity and magnetic field data as $\boldsymbol{E}=-(\boldsymbol{V} \times \boldsymbol{B})$, observed by Cl-1 between 08:20-08:25 UT. We have introduced a new coordinate system $X^{\prime} Y^{\prime} Z^{\prime}$, in which $X^{\prime}$ is directed along the mean magnetic field, $Z^{\prime}$ is orthogonal to the maximum variance direction $M: Z^{\prime}=X^{\prime} \times M$ and $Y^{\prime}$ completes the righthand coordinate system. The direction of maximum variance was determined by the MVA analysis applied to the magnetic field data measured between 08:22-08:23 UT. The projection of the new $X^{\prime} Y^{\prime} Z^{\prime}$ coordinate system to GSE system was: $X^{\prime}=[0.99,0.03,0.15] ; Y^{\prime}=[-0.06,0.83,-0.55]$, $Z^{\prime}=[-0.14,-0.55,-0.82]$. It is seen that the $B Y^{\prime}$ and $E Z^{\prime}$ components of the magnetic and electric fields experience the largest perturbation from their average values (averaged for the interval of interest and shown by the horizontal black 
a)

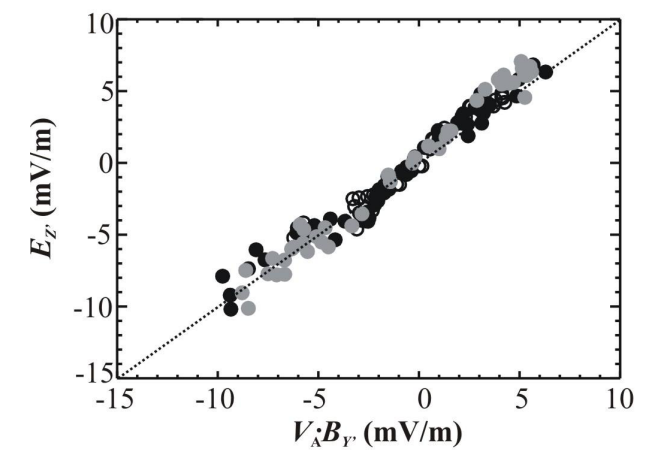

b)

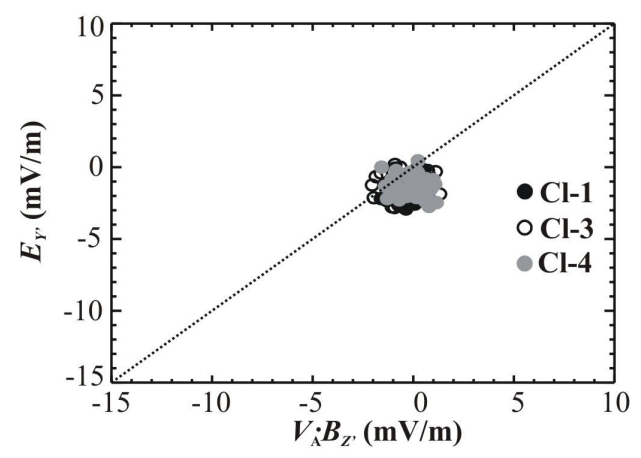

Fig. 6. Scatterplots of the values $E Z^{\prime}$ versus $V A \cdot B Y^{\prime}$ (a) and $E Y^{\prime}$ versus $V A \cdot B Z^{\prime}$ (b) obtained from the magnetic field and velocity of low-energy ions measured by three Cluster spacecraft between 08:21-08:24 UT. Alfvén velocity $V A$ was calculated from the local plasma and magnetic field parameters.

dotted lines in the Figs. 4, 5). These perturbations may be caused by the linearly polarized wave with the period $\sim 2$ min (it is shown in the Figs. 4, 5 by vertical dashed lines). The linear polarization is confirmed by the high correlation $\left(k\left(B Y^{\prime}-E Z^{\prime}\right)=0.84\right)$ between $B Y^{\prime}$ and $E Z^{\prime}$ components of the magnetic and electric fields and low correlation between $B Z^{\prime}$ and $E Y^{\prime}\left(k\left(B Z^{\prime}-E Y^{\prime}\right)=0.09\right)$. The coefficients of correlation $k\left(B Y^{\prime}-E Z^{\prime}\right)$ and $k\left(B Z^{\prime}-E Y^{\prime}\right)$ were calculated for the time interval 08:21-08:24:30 UT by the same way (see Eq. 1), as the coefficient of correlation between the perpendicular velocities of low- and high-energy ions.

Figure 6 represents two scatterplots for data obtained between 08:21-08:24 UT by three Cluster spacecraft. Plot (a) shows the dependence of $E Z^{\prime}$ variations versus $V A \cdot B Y^{\prime}$ and plot (b) displays the dependence of $E Y^{\prime}$ component versus $V A \cdot B Z^{\prime}$. The local Alfvén velocity was calculated from plasma and magnetic field parameters and was $V A \sim 900 \mathrm{~km} / \mathrm{s}$. The values of $E Z^{\prime} / B Y^{\prime}$ are ranged between $0.7 \mathrm{VA}$ and $1.5 \mathrm{VA}$, that is in good enough agreement with the Alfvén velocity calculated from the local plasma and magnetic field parameters. Taking into account that there is no linear dependence of $E Y^{\prime}\left(V A \cdot B Z^{\prime}\right)$ (see Fig. 6b), we may conclude that the wavy perturbation of the magnetic flux tubes lies in the $\left(X^{\prime} Y^{\prime}\right)$ plane and propagates along the magnetic field with the local Alfvén velocity. The wavelength of this perturbation is of the order of $16 R_{\mathrm{E}}$.

It is worth noting that, some temporal variation of the parallel velocity of high-energy ions took place in this event: the absolute value of $V_{||}$decreased from $\sim 2500 \mathrm{~km} / \mathrm{s}$, measured at the moment of Cluster entrance to the PSBL from the lobe (at $\sim 08: 21: 30 \mathrm{UT}$ ) to $\sim 2000 \mathrm{~km} / \mathrm{s}$ at the moment of Cluster exit from the PSBL to the lobe (at $\sim 08: 22: 30$ UT). There are two possible explanations of this temporal variation: 1) stopping of the acceleration process occurred in the CS tailward from the Cluster location, so ions with lower $V_{\|}$arrive the last and 2) changes of the acceleration conditions in the source (for example, local value of the magnetic field) without its stopping. The second explanation seems for us more realistic. Even when Cluster quartet was located in the southern lobe but still close to the PSBL (for example, between Cluster exit from the PSBL at $\sim 08: 23$ UT and its new encounter with the PSBL at $\sim 08: 33$ UT, not shown), periodic enhancements of energy of cold lobe ions, similar to the one observed in our interval of interest, were registered. We may assume that these energy enhancements caused by the increases of perpendicular velocity of cold lobe ions were induced by the similar perturbations of the PSBL magnetic flux tubes during the propagation of high-velocity ions in the PSBL. Thus we may assume that in this event the high-energy ions did not disappear in the PSBL when Cluster quartet exited to the lobe. We should also note that in spite of the observed temporal variation of parallel velocity of highenergy ions, its value was still two times larger than the local Alfvén velocity.

From the maximum amplitude of the magnetic and electric perturbations $\left|\delta B Y^{\prime}\right| \sim 5 \mathrm{nT},\left|\delta E Z^{\prime}\right| \sim 10 \mathrm{mV} / \mathrm{m}$, which were calculated from the average values of corresponding components observed during the interval of interest, we can estimate the Poynting flux $S_{\text {lobe }}$ as $0.04 \times 10^{-3} \mathrm{~J} / \mathrm{m}^{2} \mathrm{~s}$. Assuming that the energy flux is conserved along the magnetic tube, one may evaluate the energy flux coming to the ionosphere (mapped to the altitude $\sim 100 \mathrm{~km}$ ): $S_{\text {lobe }}$. $\left(B_{\text {iono }} / B_{\text {lobe }}\right) \sim 0.04 \mathrm{~J} / \mathrm{m}^{2}$ s. The kinetic energy flux transferred in the PSBL by the high velocity field-aligned ions is $\sim 1.1 \times 10^{-3} \mathrm{~J} / \mathrm{m}^{2}$ s, i.e. $\sim 30$ times larger than the Poynting flux carried by the wavy perturbation of PSBL magnetic flux tubes.

Summarizing all these observations, we may suggest that the propagation of high velocity ions at the lobeward edge of the PSBL can trigger Alfvén perturbations of the PSBL magnetic flux tubes. The electric field induced by these perturbations results in the transient increase of drift velocity of cold ions in the neighboring lobe region. In the next section we prove statistically that the wavy perturbation of the PSBL is 
often related to the propagation of high velocity field-aligned ions.

\section{Statistical study of the magnetic perturbations associ- ated with high velocity field-aligned ions propagating in PSBL}

In this section, we present a statistical study of magnetic perturbations detected at the PSBL-lobe interface. We have analyzed 170 PSBL crossings observed during 2001-2002 Cluster seasons in the magnetotail (at $X=-13$ to $-19 R_{\mathrm{E}}$ ). For 80 PSBL crossings neither high velocity field-aligned ions, nor transient increases of energy of cold lobe ions were observed. High velocity field-aligned ions were registered in 90 PSBL crossings. Among them, in 65 cases, the energy increases of the cold lobe ion population were also observed at the PSBL-lobe interface. The question is, whether the phenomenon of transient increases of drift velocity of cold lobe ions is always linked with the propagation of high velocity ions and the large scale wavy fluctuations of the magnetic flux tubes. We will also try to find out, what characteristics of high velocity ions propagating in the PSBL are favorable for the triggering of long wavelength perturbations, and will check statistically whether the main part of these magnetic fluctuations represents Alfvén waves.

Figure 7 shows a scatterplot of coefficients of the best correlation between electric and magnetic field variations: either between $\delta E Y^{\prime}$ and $\delta B Z^{\prime}$ or between $\delta E Z^{\prime}$ and $\delta B Y^{\prime}$ versus the correlation coefficients between the values of perpendicular velocities of low-energy (below $2 \mathrm{keV}$ ) and high-energy $(>5 \mathrm{keV}$ ) ions observed during each PSBL crossing from our data set. Coefficients of correlations were calculated using the Eq. (1). Variations of the electric and the magnetic fields were calculated in the coordinate system $X^{\prime} Y^{\prime} Z^{\prime}$ that was described in the previous section. The PSBL crossings, in which the energy increases of cold lobe ions were not observed or their densities were low $\left(<0.01 \mathrm{~cm}^{-3}\right)$, are shown by empty circles in the Fig. 7. For all PSBL crossings, in which the increases of energy of cold ions were registered, the high $(>0.7)$ correlation between the values of perpendicular velocity of high- and low-energy ions was observed. In these crossings high correlations between variations of magnetic and electric fields were also registered. The statistical analysis of PSBL magnetic perturbations, presented below, is applied only to such crossings (65 events).

Figure 8a represents a scatterplot of $\delta E Y^{\prime}$ as a function of $V A \cdot \delta B Z^{\prime}$ measured during the intervals of the PSBL crossings, when the highest correlations between $\delta E Y^{\prime}$ and $\delta B Z^{\prime}$ were observed. A scatterplot of $\delta E Z^{\prime}$ versus $V A \cdot \delta B Y^{\prime}$ is shown in Fig. $8 \mathrm{~b}$ for the PSBL crossings when the highest correlations took place between $\delta E Z^{\prime}$ and $\delta B Y^{\prime}$. The electric filed was calculated from the magnetic field and ion velocity data. Values of the Alfvén velocity $V A$ were calculated from average plasma and magnetic field characteristics observed

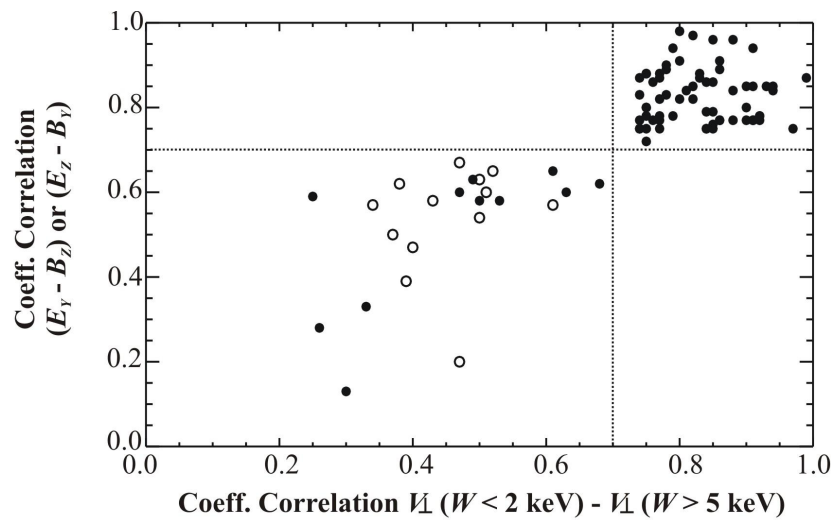

Fig. 7. Scatterplot of the coefficients of correlation between the perpendicular velocities of low-energy (below $2 \mathrm{keV}$ ) and high-energy $(>5 \mathrm{keV}$ ) ion components observed in each PSBL crossing (when high velocity field-aligned ions were registered) versus the coefficients of the best correlation between electric and magnetic field variations: either between $\delta E Y^{\prime}$ and $\delta B Z^{\prime}$ or between $\delta E Z^{\prime}$ and $\delta B Y^{\prime}$ detected in a particular crossing. The PSBL crossings, where the density of low-energy ions was $<0.01 \mathrm{~cm}^{-3}$, are shown by empty circles.

in each particular PSBL crossing. The ratios of $\delta E Y^{\prime} / \delta B Z^{\prime}$ or $\delta E Z^{\prime} / \delta B Y^{\prime}$ to the local Alfvén velocities ranged between 0.5 and 1.5. Thus, the magnetic perturbations associated with the high velocity ions, streaming along the magnetic field lines in PSBL, most likely represent Alfvén waves propagating earthward.

Figure 9a shows a scatterplot of the coefficients of correlation between $\delta B Y^{\prime}$ and $\delta E Z^{\prime}$ versus the values of the correlation between $\delta B Z^{\prime}$ and $\delta E Y^{\prime}$, for each PSBL crossing. The color of each circle represents the period of wavy perturbation of the magnetic flux tubes observed in a given PSBL crossing according to the grey scale shown at the right part of this figure. Alfvén fluctuations with the periods larger than 90 s have mostly the linear polarization. The magnetic components of these waves oscillate either along $Y^{\prime}$ or along $Z^{\prime}$ directions with approximately equal probability. Alfvén fluctuations with short enough periods $(T<90 \mathrm{~s})$ have mostly elliptically (left-hand) polarization. The region occupied by these events is shaded by the grey color in Fig. 9a. In Fig. $9 \mathrm{~b}$ the coefficients of correlation between $\delta E Y^{\prime}(t)$ and $\delta E Z^{\prime}(t+T / 4)$ versus the coefficients of correlation between $\delta B Y^{\prime}(t)$ and $\delta B Z^{\prime}(t+T / 4)$ are shown for the elliptically polarized perturbations. Variations of $Z^{\prime}$-components of the electric and magnetic field observed in each event were timelagged at $T / 4$, where $T$ is a period of the particular magnetic fluctuations. The elliptical polarization of the waves with $T<90 \mathrm{~s}$ observed in our data set conforms to the previous observations of Alfvén waves in the magnetotail (Sauvaud et al., 2004).

The statistical distributions of periods and wavelengths of the Alfvén fluctuations observed in PSBL during the 

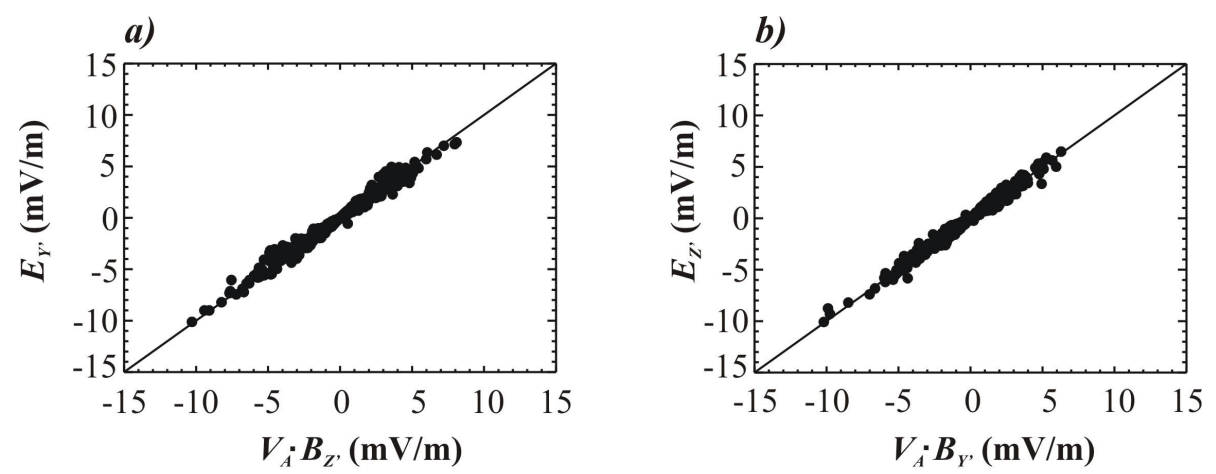

Fig. 8. (a) Scatterplot of $\delta E Y^{\prime}$ versus $V A \cdot \delta B Z^{\prime}$ measured during the intervals of PSBL crossings, when the strongest correlation between $E Y^{\prime}$ and $B Z^{\prime}$ components was observed. (b) Scatterplot of $\delta E Z^{\prime}$ versus $V A \cdot \delta B Y^{\prime}$ is presented for the PSBL crossings in which the highest correlations between $E Z^{\prime}$ and $B Y^{\prime}$ components were registered.

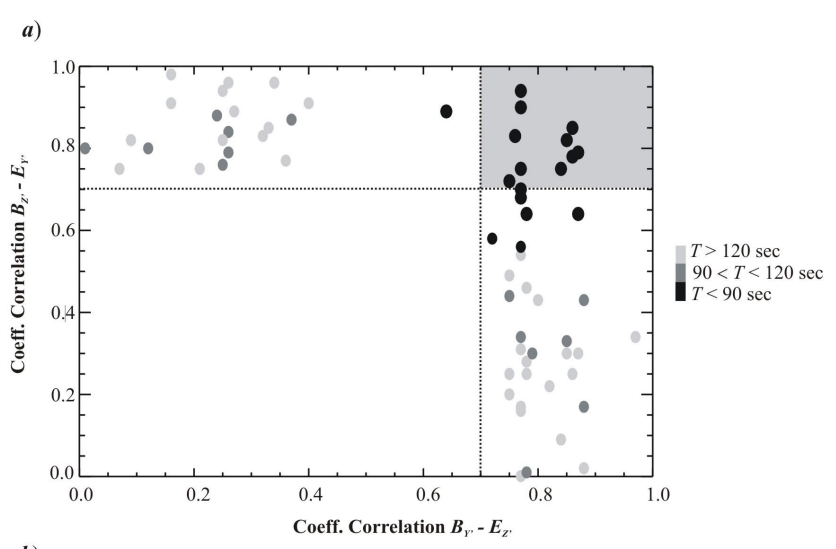

b)

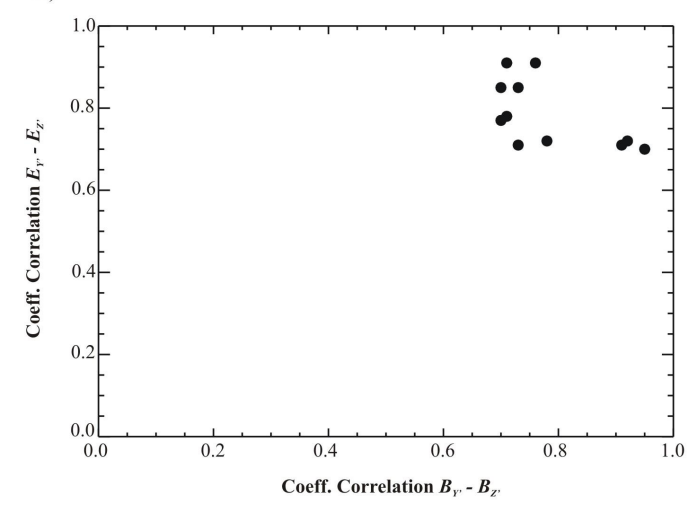

Fig. 9. (a) Scatterplot of the coefficients of correlation between $\delta B Y^{\prime}$ and $\delta E Z^{\prime}$ versus the values of the correlation between $\delta B Z^{\prime}$ and $\delta E Y^{\prime}$, for those PSBL crossings, where high $(>0.7)$ correlations between drift velocities of high- and low-energy ions were detected. The color of each circle represents the period of wavy perturbation of the magnetic flux tubes observed in a given PSBL crossing according to the grey scale shown at the right part of this figure. (b) Scatterplot of the coefficients of correlation between $\delta E Y^{\prime}(t)$ and $\delta E Z^{\prime}(t+T / 4)$ versus the coefficients of correlation between $\delta B Y^{\prime}(t)$ and $\delta B Z^{\prime}(t+T / 4)$ for the circularly polarized perturbations. intervals of propagation of high velocity field-aligned ions compiled from our data set are shown in Fig. 10a, b. Wavelengths were calculated for each crossing as $L=T \cdot V A$, where $V A$ was the local Alfvén velocity deduced from the plasma and magnetic field parameters. The majority of the waves have periods $\sim 1-4 \mathrm{~min}$ and wavelengths in the range $L \sim 5-20 R_{\mathrm{E}}$.

The question, whether high velocity field-aligned ions registered in the PSBL represent a beam or they are a component of high velocity plasma flow, seems to us very important. We have compared the values of total field-aligned current $\left(J_{\|}^{\text {curl }}\right)$ calculated in each PSBL crossing as $(\nabla \times \boldsymbol{B}) \|$ with the field-aligned component of ion current $J_{\|}^{\text {ion }}=e n V \|$. For each interval of interest a ratio between $(\nabla \cdot \boldsymbol{B})$ and $(\nabla \times \boldsymbol{B})$ was less than 0.2. A scatterplot of the ratio of total fieldaligned current to ion field-aligned current versus the values of ion field-aligned current is presented in Fig. 11. In the majority of cases when ion parallel velocities exceeded double value of local Alfvén velocity (these events are displayed by black dots) the total field-aligned current $J_{\|}^{\text {curl }}$ was significantly smaller than the field-aligned current produced by high velocity ions. This means that in these PSBL crossings ion currents were compensated by field-aligned electron currents and the high velocity plasma flows rather than beams propagate towards the Earth in the PSBL-lobe interface.

\section{Kelvin-Helmholtz instability in a bounded flow}

It is well known that a propagation of high-energy ions could serve as a source of free energy for different types of plasma instabilities. As follows from the analysis of experimental data presented above, typical electromagnetic fluctuations of the PSBL magnetic flux tubes under our study have very long wavelengths ( $\lambda \approx 5-20 R_{\mathrm{E}}$ ) and ultra-low frequencies ( $f \approx 0.004-0.02 \mathrm{~Hz}$ ) significantly below the local proton gyrofrequency. The typical phase velocity of these fluctuations is of the order of local Alfvén velocity, which is more than 

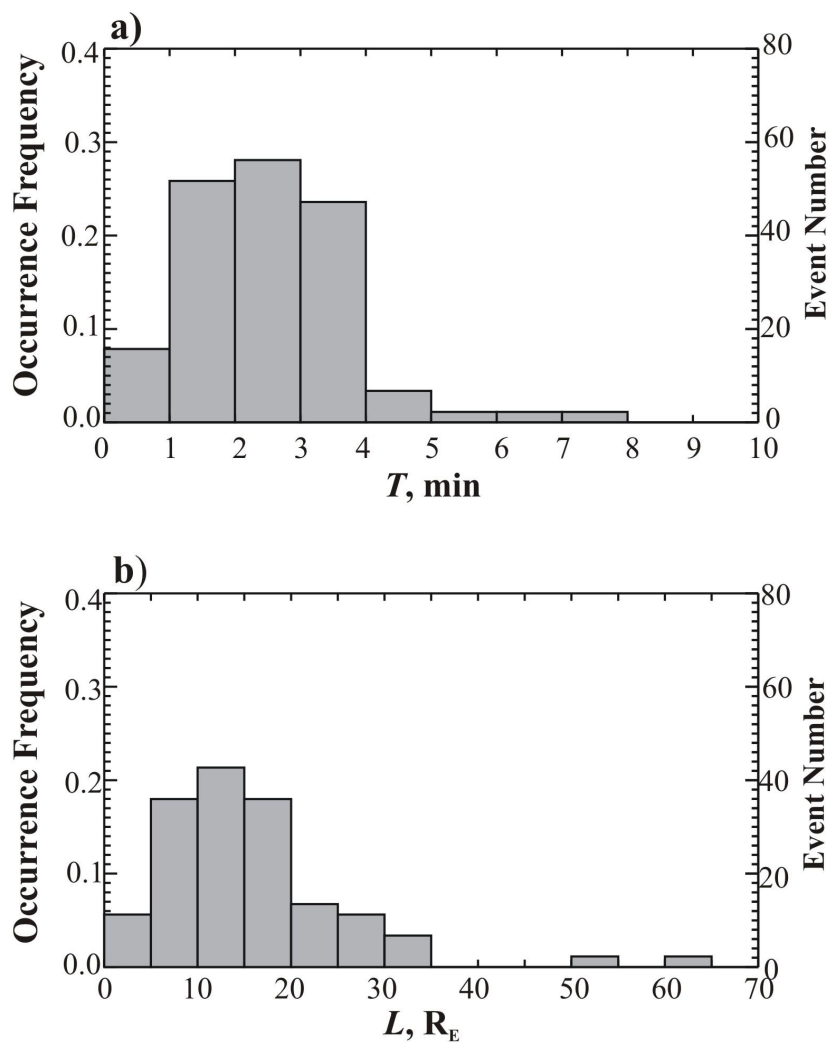

Fig. 10. Statistical distributions of wavy fluctuations associated with high velocity field-aligned ions: (a) periods $T$ and (b) wavelengths $L$.

twice below the measured ion flow speed in the most cases considered in our study. The generation of this type of oscillations can be caused by a development of the currentdriven kink-like instability (Angelopoulos et al., 1989; Gary and Winske, 1990) or the Kelvin-Helmholtz (KH) instability (Sauvaud et al., 2004). Since in the most part of the PSBL crossings analyzed in the present study the currents, corresponding to electron-ion relative drift, are very low (see Fig. 11), the excitation of the current-driven instability is impossible. Thus, the K-H instability triggered by a strong velocity gradient, appearing at the lobe-PSBL and PSBLCPS interfaces during the propagation of plasma flows in the PSBL, seems the most likely candidate for the generation of the observed wavy perturbations of magnetic flux tubes.

There are two important points in the investigation of the $\mathrm{K}-\mathrm{H}$ instability in the PSBL. First, as the PSBL lies between the hot, dense PS and tenuous cold lobe plasma, it is important to include the variations of plasma parameters around the flux tube with high-energy ions. Second, since the characteristic wavelengths of fluctuations under study are far in excess of typical plasma flow dimensions in the plane perpendicular to the background magnetic field, the K-H instability should be studied in a plasma flow confined in the plane perpendicular to the magnetic field. The investigation of the K-H in-

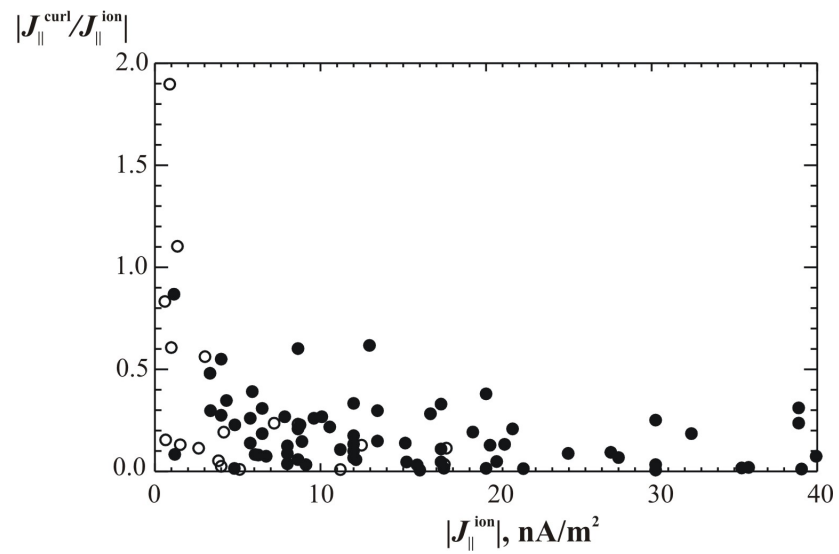

Fig. 11. Scatterplot of absolute values of the ratio of total current density $(\nabla \times B) \|$ to the density of field-aligned current produced by high velocity ions $\left(J_{\|}^{\text {curl }} / J_{\|}^{\text {ion }}\right)$ versus the absolute values of density of ion field-aligned current ( $\left.J_{\|}^{\text {ion }}\right)$ measured in each PSBL crossing from our data set. The PSBL crossings in which the parallel velocity of high-energy ions was less than the double value of local Alfvén velocity are displayed by empty dots.

stability, taking into consideration both points, needs a rather complicated 3-D simulation even at the linear stage of the instability.

So, with the purpose to evaluate roughly the possible role of the K-H instability in the generation of electromagnetic fluctuations under study, we use a simplified slab model, where the plasma flow has a finite width $2 a$ only in Z-direction and is unlimited in X- and Y-directions (see Fig. 12). The background magnetic field $\boldsymbol{B}_{0}$, both inside and outside the flow, as well as the flow velocity $\boldsymbol{U}_{0}$, is directed along the $\mathrm{X}$-axis. The plasma component surrounding the flow $(Z>a, Z<-a)$ is at rest and may have different parameters at both sides of the flow. A thickness of the transition shear layers between a flow and surrounding plasma is supposed to be much smaller than a flow width.

The general dispersion equation for the stability of the MHD slab equilibrium is derived with a help of a set of ideal MHD equations for each of the considered plasma regions ( $Z<-a,-a<Z<a, Z>a$ ) and by using the total (gaskinetic and magnetic) pressure balance condition, and the continuity of a boundary displacement in the Z-direction at the interfaces between the flow and surrounding plasma. The obtained dispersion equation for eigenmodes vanishing at the infinity may be written as

$\left(k_{f}^{2} R_{1} R_{2}+k_{1} k_{2} R_{f}^{2}\right) \tanh \left(2 k_{f} a\right)+k_{f} R_{f}\left(k_{1} R_{2}+k_{2} R_{1}\right)=0,(2)$

where

$k_{f}^{2}=$

$k^{2}\left(1-\frac{\left(\omega / \boldsymbol{k} \boldsymbol{U}_{0}-\cos \theta\right)^{4}}{\left(\omega / \boldsymbol{k} \boldsymbol{U}_{0}-\cos \theta\right)^{2}\left(v_{\mathrm{A} f}^{2}+c_{\mathrm{S} f}^{2}\right) / \boldsymbol{U}_{0}^{2}-v_{\mathrm{A} f}^{2} c_{\mathrm{S} f}^{2} \cos ^{2} \theta / \boldsymbol{U}_{0}^{4}}\right) ;$ 


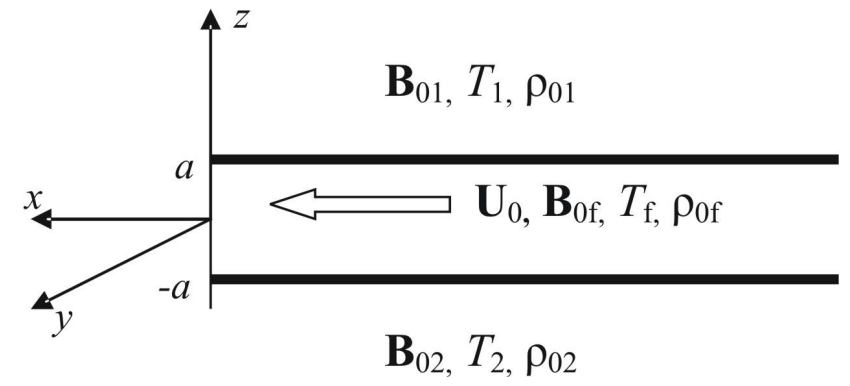

Fig. 12. Geometry of the three-layer model.

$k_{j}^{2}=k^{2}\left(1-\frac{\left(\omega / \boldsymbol{k} \boldsymbol{U}_{0}\right)^{4}}{\left(\omega / \boldsymbol{k} \boldsymbol{U}_{0}\right)^{2}\left(v_{\mathrm{A} j}^{2}+c_{\mathrm{S} j}^{2}\right) / \boldsymbol{U}_{0}^{2}-v_{\mathrm{A} j}^{2} c_{\mathrm{S}_{j}}^{2} \cos ^{2} \theta / \boldsymbol{U}_{0}^{4}}\right)$, $j=1,2$;

$R_{f}=\left(\omega / \boldsymbol{k} \boldsymbol{U}_{0}-\cos \theta\right)^{2}-\frac{\boldsymbol{B}_{0 f}^{2} \cos ^{2} \theta}{4 \pi \rho_{0 f} \boldsymbol{U}_{0}^{2}} ;$

$R_{j}=\frac{\rho_{0 j}}{\rho_{0 f}} \frac{\omega^{2}}{k^{2} \boldsymbol{U}_{0}^{2}}-\frac{\boldsymbol{B}_{0 j}^{2} \cos ^{2} \theta}{4 \pi \rho_{0 f} \boldsymbol{U}_{0}^{2}}, \quad j=1,2$.

Here the index $f$ is related to the parameters of the flow region and $j=1,2$ to the plasma parameters outside the flow, $\rho_{0}$ is a background plasma mass density, $c_{\mathrm{S}}$ is the ion sound velocity, $v_{\mathrm{A}}$ is the Alfvén velocity and $\theta$ is an angle between a wave vector $\boldsymbol{k}$ and a background magnetic field $\boldsymbol{B}_{0}$.

It is well known that at the interface between two semiinfinite compressible plasmas with a relative motion along the magnetic field, oscillations with wave vectors, lying close enough to the magnetic field direction $\left(0 \leq \theta \leq \theta_{\max }\right.$, where $\theta_{\max }$ depends on the plasma parameters), can be driven by the $\mathrm{K}-\mathrm{H}$ instability only when the plasma temperature is high enough, namely, when $c_{\mathrm{S}}>v_{\mathrm{A}}$ (Blumen, 1970; Miura and Pritchett, 1982). Taking into account the finite thickness of a flow in a three-layered system leads to the possibility of the development of the K-H instability for the long wavelength oscillations even at zero temperature $\left(c_{S}=0\right)$. There are two types of solutions of the obtained dispersion Eq. (2): the kink and the sausage-like modes. Under low-temperature conditions typical for the PSBL the growth rate of the kink-like mode is much larger than the one for the sausage-like mode (Burinskaya, 2008; Burinskaya and Shevelev, 2010).

Figure 13 displays the growth rate of the kink-like modes normalized to $a / \boldsymbol{U}_{0}$ as a function of $k a$ for various angles $\theta$ of wave propagation. Figure $13 \mathrm{~b}$ shows the polar plots of the normalized growth rate $\gamma a / \boldsymbol{U}_{0}$ as a function of $\theta$ for the fixed values of $k a$. Solid curves represent the growth rates calculated by the Eq. (2) for $k a=0.5 ; 1.0 ; 1.5$. Dashed and dot-and-dash curves are shown for a common case of two semi-infinite plasmas for $k a=0.5$. Dashed curves relate to the development of the K-H instability at the interface between the flow and region 1, and dot-and-dash lines describe the same process at the interface between the flow
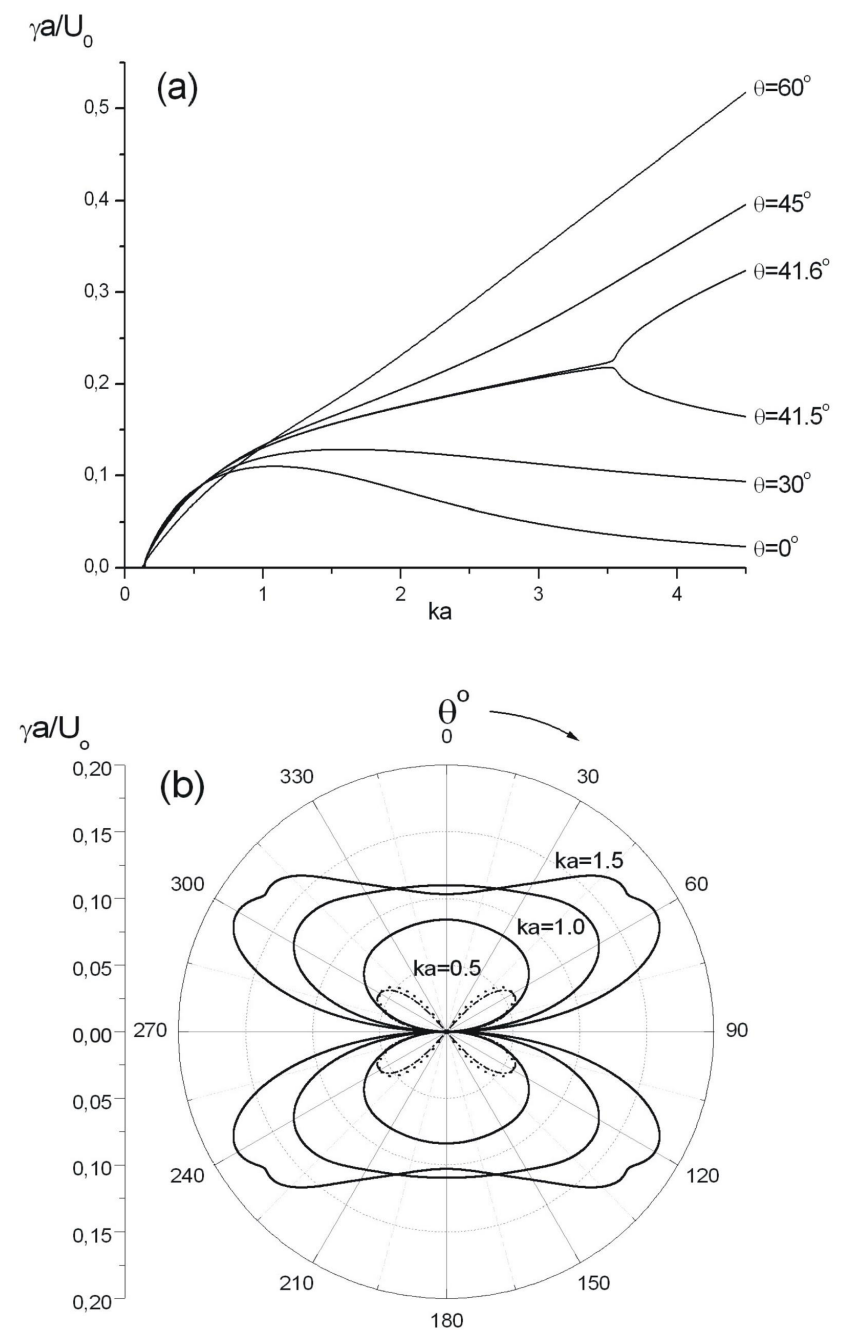

Fig. 13. (a) The growth rate $\gamma a / \boldsymbol{U}_{0}$ as a function of $k a$ for various angles $\theta$ of wave propagation. Plasma densities in all three regions are taken different $\rho_{01} / \rho_{0 f}=0.8, \rho_{02} / \rho_{0 f}=1.2$. Other parameters are $c_{S 1}^{2} / \boldsymbol{U}_{0}^{2}=0.001, c_{s 2}^{2} / \boldsymbol{U}_{0}^{2}=c_{\mathrm{S} f}^{2} / \boldsymbol{U}_{0}^{2}=0.01$ and $v_{\mathrm{A} f} / \boldsymbol{U}_{0}=0.316$; (b) Polar plots of the normalized growth rate $\gamma a / \boldsymbol{U}_{0}$ as a function of $\theta$ for fixed $k a$. Solid curves represent the growth rates calculated for the three-layered plasma. Dashed and dot-and-dash curves show $\gamma a / \boldsymbol{U}_{0}$ calculated for two semi-infinite plasmas. Dashed curves are related to the boundary between the region 1 and flow, and dot-and-dashed lines - to the boundary between the region 2 and flow.

and region 2. The results presented in Fig. 13 are obtained for the case when plasma densities in all three regions are different $\rho_{01} / \rho_{0 f}=0.8, \rho_{02} / \rho_{0 f}=1.2$ and $c_{S 1}^{2} / \boldsymbol{U}_{0}^{2}=0.001$, $c_{s 2}^{2} / \boldsymbol{U}_{0}^{2}=c_{\mathrm{S} f}^{2} / U_{0}^{2}=0.01$. The ratio of the Alfvén velocity in the PSBL to the flow velocity is taken $v_{\mathrm{A} f} / \boldsymbol{U}_{0}=0.316$ and, as it follows from the pressure balance for the regions 1 and $2, v_{A 1} / \boldsymbol{U}_{0}=0.373$ and $v_{A 2} / \boldsymbol{U}_{0}=0.285$.

It is clearly seen that in a three-layered plasma long wavelength fluctuations exist for all angles $\theta$ (except $\theta=90^{\circ}$, as it 
has to be) contrary to the case of two semi-infinite plasmas, where waves can grow only in the range $35.2^{\circ \circ}<\theta<90^{\circ}$ for the lobe-PSBL interface and in the range $41.5^{\circ \circ}<\theta<90^{\circ}$ for the PSBL-CPS interface, see Fig. 13b. It should be noted that the growth rate of long wavelength oscillations with $k a<1$ is always greater for the case of a three-layered plasma, than for the common case of two semi-infinite plasmas. It follows from Fig. 13a that the long wavelength fluctuations generated at rather small angles around the background magnetic field, are developed in the limited band of unstable wave numbers. The growth rate of oscillations excited at large enough angles around the magnetic field linearly increases with a wave number. However, our results are valid for the oscillations with wavelengths well greater than the thickness $\Delta$ of a shear layer between the flow and surrounding plasma, $k \Delta \ll 1$. As it was assumed that $a \gg \Delta$, we may use the obtained results as a close approximation for wavelength long enough.

Figure 14 shows displacements $\xi$ of the plasma flow boundaries in the z-direction in dimensionless units as a function of the $\mathrm{x}$-coordinate normalized to the half width of the flow. The solid and dashed lines represent the displacements of the boundaries between the plasma flow and the regions 1 and 2, correspondingly. For the convenience of comparison, the profiles of both boundaries are superposed. The results displayed in Fig. 14a are obtained for the same parameters as in Fig. 13. Displacements $\xi_{1}$ and $\xi_{2}$, arising due to the wave propagation when $k a=1$ and $\theta=30^{\circ}$, are shown in Fig. 14a. In this case the flow velocity $\boldsymbol{U}_{0}$ is well above the sum of the Alfvén velocities in the flow region and in the ambient plasma from both sides of the plasma flow, that is $U_{0}>v_{A 1}+v_{\mathrm{A} f}$ and $\boldsymbol{U}_{0}>v_{A 2}+v_{\mathrm{A} f}$. It is evident from the Fig. 14a that the amplitude of the displacement of the boundary between the plasma flow and region 1 (imitating the lobe region) is slightly larger than that of the other boundary between the plasma flow and the region 2 (imitating the CPS).

The results shown in Fig. 14b are calculated for the same plasma densities and temperatures as the ones presented in Fig. 13, but the ratio of the Alfvén velocity in the PSBL to the flow speed is taken as $v_{\mathrm{A} f} / \boldsymbol{U}_{0}=0.474$. In the regions 1 and 2 we have from the pressure balance $v_{A 1} / \boldsymbol{U}_{0}=0.543$, $v_{A 2} / \boldsymbol{U}_{0}=0.431$, correspondingly. In such a way, the flow speed is taken to be greater than the sum of the Alfvén velocities in the PSBL and in the CPS, but less than the sum of the Alfvén velocities in the PSBL and in the lobe. In this case, as shown in Fig. 14b, the amplitude of the displacement of the boundary between the flow and the region 2 (the CPS) is slightly larger than that of the other boundary, contrary to the case shown in Fig. 14a. One can see from Fig. 14a, b that the wave phases at both sides of the flow differ only slightly, i.e., the flow oscillations resemble the kink-like waves. It is important to stress that a three-layered plasma could be unstable against the excitation of long wavelength perturbations, when the flow speed at one of the boundaries is less than the sum of the Alfvén velocities in the flow and
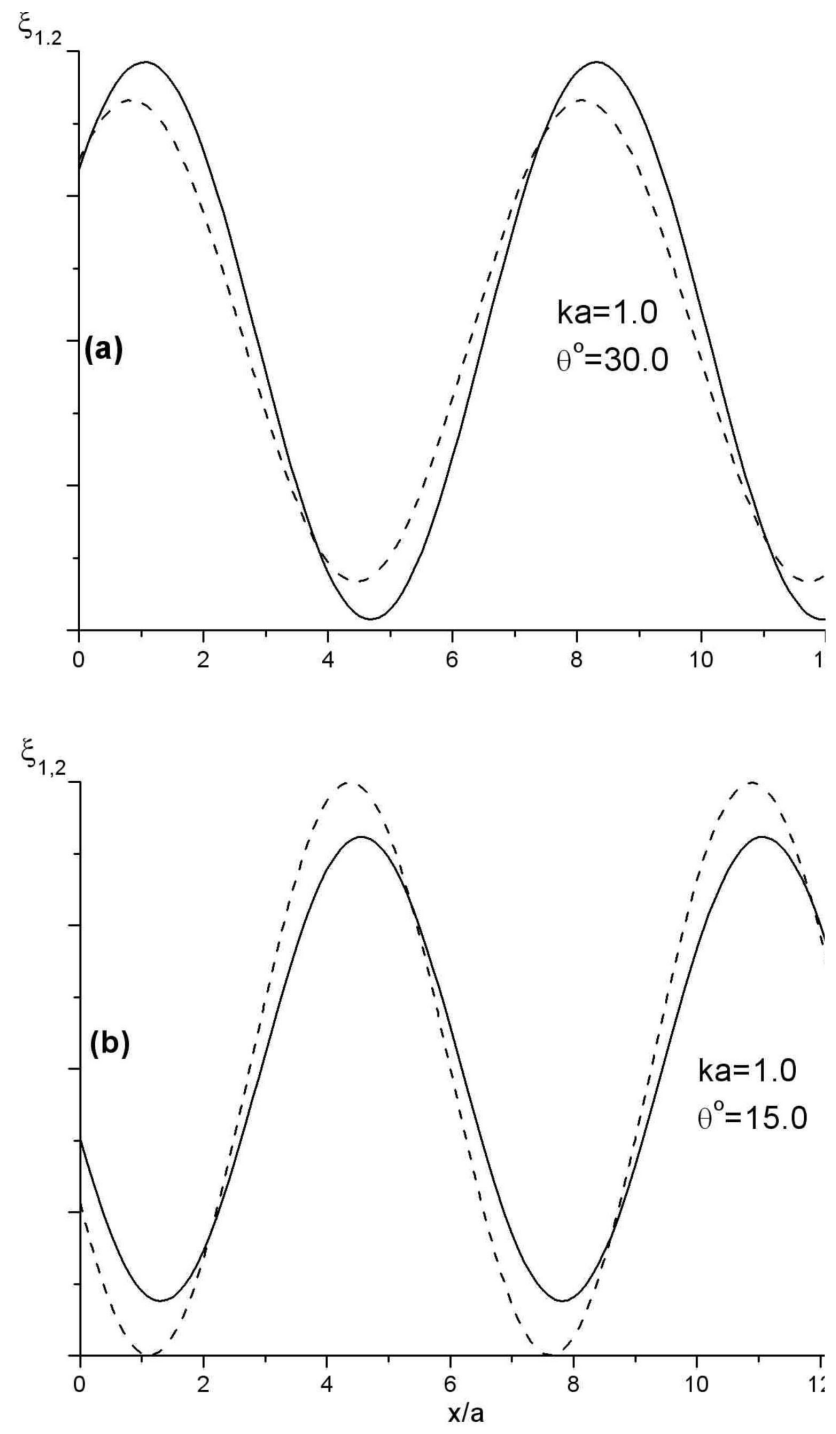

Fig. 14. Displacements of plasma flow boundaries in z-direction are shown in dimensionless units as a function of $x / a$. The displacement $\xi_{1}$ (solid line) is related to the interface between the plasma flow and the region 1 (the lobe), and $\xi_{2}$ (dashed line) is related to the interface between the plasma flow and the region 2 (the CPS). The profiles of both displacements are superimposed. Plasma densities and temperatures are the same as in Fig. 13 and (a) $v_{\mathrm{A} f} / \boldsymbol{U}_{0}=0.316$; (b) $v_{\mathrm{A} f} / \boldsymbol{U}_{0}=0.474$.

the ambient plasma. Thus, a spacecraft crossing the lobePSBL interface could observe magnetic flux tube perturbations driven by the K-H instability, even when the common criterion $\boldsymbol{U}_{0}>v_{\mathrm{A} j}+v_{\mathrm{A} f},(j=1,2)$ is not fulfilled at the lobe-PSBL interface, but is true for the PSBL-CPS interface. 


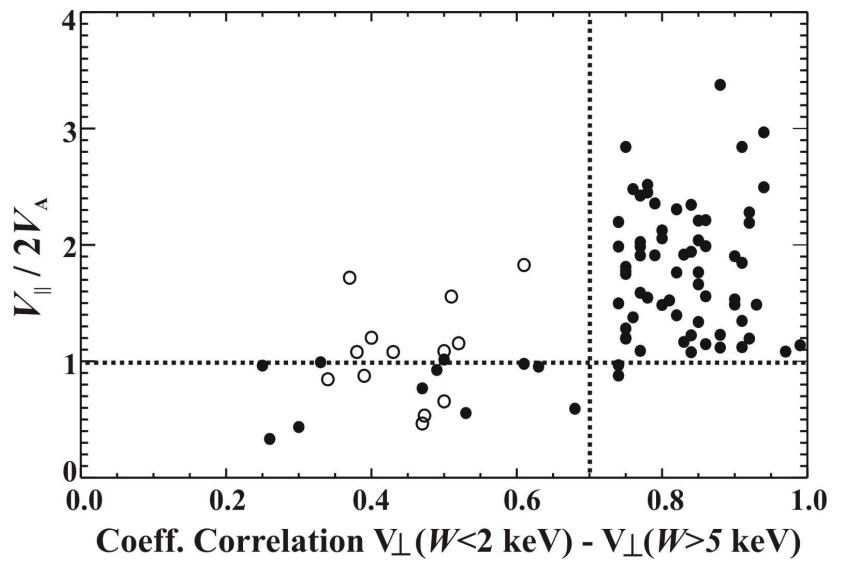

Fig. 15. Scatterplot of the parallel velocities of high-energy ions normalized to the double value of the local Alfvén velocity, calculated for each PSBL crossing, versus the coefficients of correlation between the perpendicular velocities of high- and low-energy ions. The PSBL crossings, where the density of low-energy ions was $<0.01 \mathrm{~cm}^{-3}$, are shown by empty circles.

\section{Discussion}

The phenomenon of sudden increases of drift velocities of cold lobe ions near the lobeward edge of the PSBL was reported by many authors (e.g. Sauvaud et al., 2004; Zelenyi et al., 2004; Keiling et al., 2006; Grigorenko et al., 2007). They linked this feature with the wavy perturbation of the PSBL boundary arising due to the propagation of a high velocity field-aligned ion beam. One of the most important objects of present study was to check statistically the causal relationship between the appearance of high velocity ions, propagating along the magnetic field lines at the lobeward edge of the PSBL, and transient increases of drift velocity of low-energy lobe ions observed in the lobe-PSBL interface.

In our data set, the transient increases of energy of cold lobe ion population up to $\geq 1 \mathrm{keV}$ were registered in $72 \%$ of PSBL crossings. During all these intervals high velocity field-aligned ions were also observed at the lobeward edge of the PSBL. First of all, we would like to stress that in the majority of these PSBL crossings high velocity field-aligned ions represented a component of high velocity plasma flows. We made this conclusion from the comparative analysis of the values of field-aligned current density calculated from $(\nabla \times \boldsymbol{B}) \|$ and the values of ion field-aligned current density estimated from the density and parallel velocity of high - energy ions. For the most events from our data set ion fieldaligned current was directed in the same direction as the total field-aligned current $(\nabla \times \boldsymbol{B}) \|$. However the total current, corresponding to electron-ion relative drift, was significantly smaller than the ion current. It means that for these events the ion field-aligned current was almost compensated by the field-aligned component of the electron current, i.e. ions and electrons moved together as a flow.
For all PSBL crossings, when the energy increases of cold ion population were registered, a high correlation $(\geq 0.7)$ between the perpendicular velocities of high-energy ions and low-energy lobe ions was found. This indicates the presence of a perturbation of the magnetic flux tubes, containing high velocity ions and low-energy lobe ions, in the direction perpendicular to the local magnetic field. In Sect. 3 we have statistically shown that such perturbations had a wavy nature and propagated along the magnetic field lines approximately with local Alfvén velocity. Their characteristic periods and wavelengths are $\sim 1-4$ min and $\sim 5-20 R_{\mathrm{E}}$, correspondingly.

The ultra-low frequency values of these wavy perturbations (much less than the ion gyrofrequency in the PSBL) and the fact that in the majority of cases from our data set the currents, corresponding to electron-ion relative drift, are very low, it is possible to consider the K-H instability as the most likely mechanism for the generation of observed fluctuations. The linear stage of the $\mathrm{K}-\mathrm{H}$ instability was investigated in a bounded flow sandwiched between the CPS and the lobe with plasma and magnetic field characteristics typical for these regions. It was shown that a finite thickness of a bounded flow plays a key role in the generation of oscillations with wavelengths comparable or far in excess of flow thickness propagating along the magnetic field. Assuming that $a \approx 0.5 R_{\mathrm{E}}$, which is a reasonable value for the half-width of the PSBL along the south-north direction (e.g. Takahashi and Hones, 1988; Grigorenko et al., 2007), the wavelengths $L$, corresponding to the range of full width half maximum of the linear growth rate of K-H instability calculated for different plasma parameters, fall into the interval: $1 R_{\mathrm{E}}<L<16 R_{\mathrm{E}}$. This is in an agreement with the results of our statistical analysis (see Fig. 10b), from which follows that typical wavelengths of the PSBL Alfvén fluctuations are of the order of 5-20 $R_{\mathrm{E}}$.

It was found that the K-H instability can be excited in the PSBL even when the common criterion of the K-H instability (i.e., a field-aligned velocity of plasma flow exceeds the sum of Alfvén velocities in the PSBL and in the surrounding plasma) is fulfilled only at one of the PSBL boundaries (either at the lobeward edge or at the boundary with the PS region). In Fig. 15 the ratio $V \| /(2 V A)$, where $V \|$ is a field-aligned velocity of high-energy ions and $V \mathrm{~A}$ is a local Alfvén velocity, is shown versus the values of coefficients of correlation between the perpendicular velocities of high$(>5 \mathrm{keV})$ and low-energy $(<2 \mathrm{keV})$ ion components calculated for each of 90 PSBL crossings from our data set. Since the Alfvén velocity decreases towards the neutral sheet, we assume that the value of the Alfvén velocity in the PSBL could be considered as an average between the values of Alfvén velocities in the lobe and in the PS. In the majority of the PSBL crossings, where field-aligned velocity of high energy ions exceeded double value of local Alfvén velocity, the high correlation $(\geq 0.7)$ between the drift velocities of low and high-energy ions was also registered. For the same PSBL crossings ultra-low frequency waves propagating 
with approximately Alfvén velocities were detected. Thus all these events may be described by the following scenario: i) the K-H instability is excited when the plasma flow velocity is high enough $(V \|>2 V A)$; ii) the ultra-low frequency electromagnetic oscillations are generated and propagate along the magnetic field approximately with the Alfvén velocity; iii) the low and high-energy ions acquire the same drift velocity $V=(\boldsymbol{E} \times \boldsymbol{B}) / B^{2}$, where $\boldsymbol{E}$ is the wave electric field and $\boldsymbol{B}$ is the background magnetic field.

There are few PSBL crossings where the parallel velocities of high-energy ions were less than $2 V A$, but the correlations between perpendicular velocities of low and high-energy ion components were high, and the other signatures of wavy perturbations of the magnetic flux tubes were also registered. For such cases, we may suggest two possible explanations: either the local Alfvén velocity was overestimated because of underestimation of $\mathrm{O}^{+}$ion density or the source of perturbation was located deeper in the PS.

A number of events with low $(<0.7)$ correlations between the perpendicular velocities of low- and high-energy ions were also detected. They include the PSBL crossings in which the density of low-energy ions was too low for a reliable velocity determination $\left(n<0.01 \mathrm{~cm}^{-3}\right)$. These events are shown by empty circles in Fig. 15. In the other events, where the densities of low-energy ions were high enough $n>0.01 \mathrm{~cm}^{-3}$, the field-aligned velocities of high-energy ions $V \|$ were less than or equal to $2 V A$. During such intervals we did not find a good correlation between the fluctuations of the electric and magnetic fields associated with the propagation of ultra-low frequency Alfvén wave.

Surely, it would be very interesting to observe directly the geometry of these perturbations. There are several cases then Cluster observations in the PSBL have shown that the magnetic flux tube containing high velocity ions has a crosssection comparable with Cluster separation. One of such unique cases was registered on 21 September 2001 and analyzed by Grigorenko et al. (2007). Authors reconstructed the 2-D spatial structure of the oscillating flux tube and estimated the period of this perturbation $\sim 4 \mathrm{~min}$. Unfortunately, a value of local Alfvén velocity, as well as a characteristic wavelength, was overestimated, because the density of oxygen ions was not considered. Taking into account oxygen density, the wavelength of this perturbation must be estimated as $\sim 20 R_{\mathrm{E}}$. The theoretical consideration of the generation of the long wavelength oscillations presented in this paper should be considered as a first step in investigation of the real plasma system since it does not take into account all observation features, namely, time-dependent processes and a finite cross-section of the magnetic flux tube with highvelocity plasma flow. This will be a subject of our future publication. In this paper we just want to estimate the possibility of the generation of the observed large scale fluctuations propagating along the magnetic field in the PSBL plasma, where ion sound velocity $C s$ is significantly smaller than the local $V_{\mathrm{A}}$, due to development of the K-H instability.
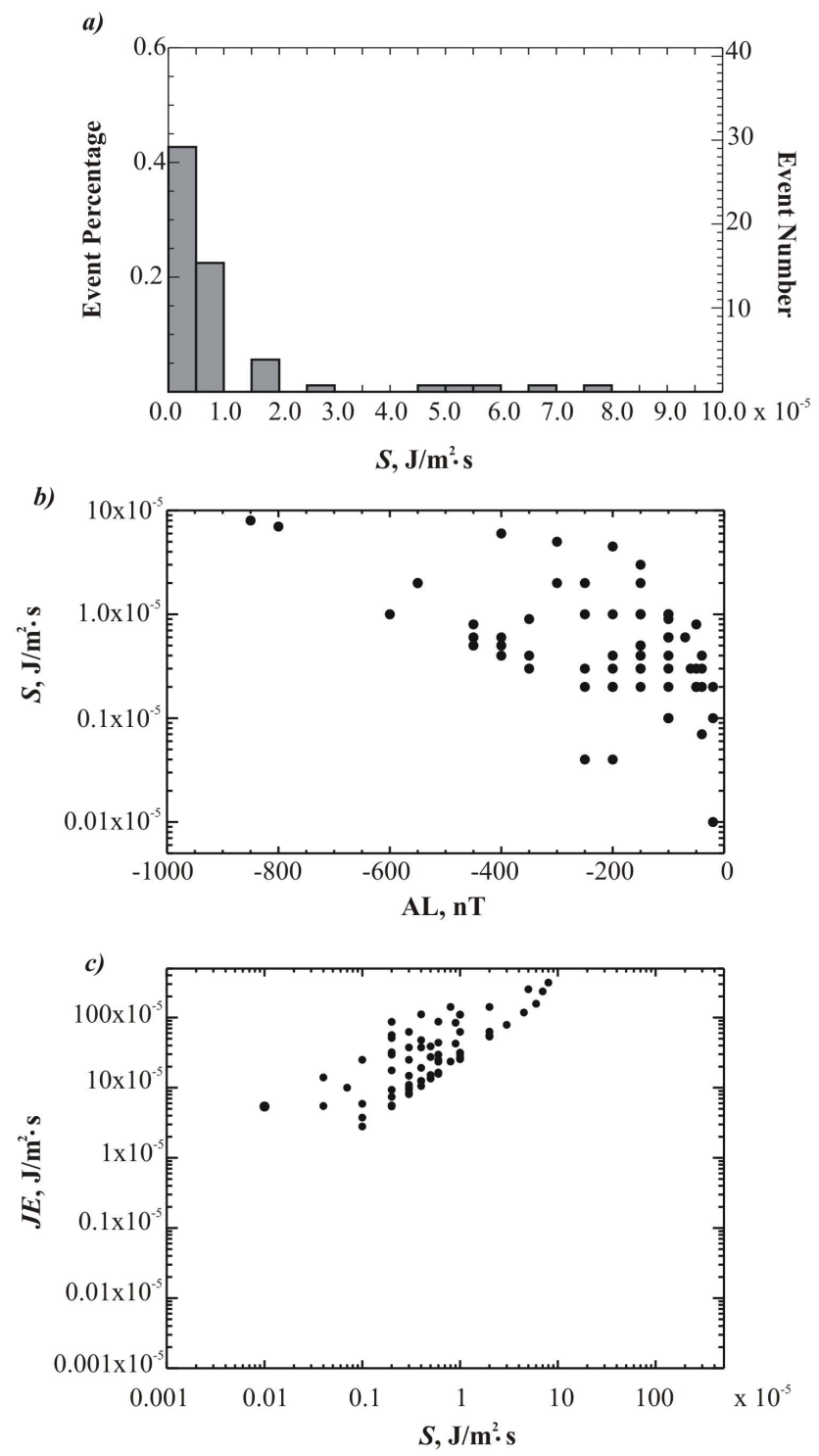

Fig. 16. (a) Statistical distribution of values of the Poynting flux, $S$ observed during the intervals of the electromagnetic perturbations associated with high velocity field-aligned ions. (b) Scatterplot of the Poynting flux versus AL index. (c) Scatterplot of kinetic energy flux $J E$, transferred by high velocity field-aligned ions versus the corresponding values of the Poynting flux.

This possibility was under question because when $C s<V_{\mathrm{A}}$ it is impossible to excite waves propagating along the magnetic filed in the common case of two plasmas.

In the PSBL and the lobe the earthward moving Alfvén waves with characteristic periods of the order of 40-300 s were also registered by the Polar spacecraft at geocentric distances from 4 to $7 R_{\mathrm{E}}$ (Keiling et al., 2000, 2001, 2005, 2009; Wygant et al., 2000). The authors have shown that during active periods, in particular, during the expansion phase of a substorm, these waves may transfer a significant amount of energy up to $2.5 \times 10^{-3} \mathrm{~J} / \mathrm{m}^{2} \mathrm{~s}$. When 
mapped to the ionospheric altitudes along the converging magnetic field lines, the Poynting flux corresponded to values above $0.1 \mathrm{~J} / \mathrm{m}^{2} \mathrm{~s}$. Figure 16 presents a statistical distribution of the Poynting flux values, which were calculated from the amplitudes of electric and magnetic field fluctuations detected in each PSBL crossing under our study. We have shown that the typical values of the Poynting flux directed towards the Earth were rather small: $S_{\text {lobe }} \leq$ $0.01 \times 10^{-3} \mathrm{~J} / \mathrm{m}^{2} \mathrm{~s}$. In these cases the corresponding flux deposited to the ionosphere (mapped to the altitude $\sim 100 \mathrm{~km}$ ) $S_{\text {iono }}=S_{\text {lobe }} \cdot\left(B_{\text {iono }} / B_{\text {lobe }}\right) \leq 0.01 \mathrm{~J} / \mathrm{m}^{2} \mathrm{~s}$. For several events the Poynting flux larger than $0.05 \times 10^{-3} \mathrm{~J} / \mathrm{m}^{2} \mathrm{~s}$ was registered and so, the value of the Poynting flux deposited to the ionosphere was $>0.05 \mathrm{~J} / \mathrm{m}^{2} \mathrm{~s}$. These observations conform to the earlier Polar observations during the substorm periods (e.g. Wygant et al., 2000; Keiling et al., 2000, 2001). It is worth noting that we did not find the unique dependence of the Poynting flux transferred by the ultra-low frequency Alfvén waves in the PSBL on the value of the AL index (see a scatterplot presented in Fig. 16b). The events with both very small and large values of the Poynting flux were observed in the rather wide range of $\mathrm{AL}$ values $(-500$ to $-20 \mathrm{nT})$.

It is also worth comparing the flux of kinetic energy transferred in the PSBL by high velocity field-aligned ions $J E=$ $\frac{n m V_{\|}^{3}}{2}$ (where $n$ is a number density of high velocity ions, $m$ is the proton mass and $V \|$ is a field-aligned ion velocity) with the values of the Poynting flux, transferred by the Alfvén waves. From the scatterplot of $J E(S)$ presented in Fig. 16c it follows that the values of the kinetic energy flux transferred by high velocity ions in the PSBL are in the range 30-500 times higher than the values of the corresponding Poynting flux. This means that the energy loss due to the transformation of the ion kinetic energy into the electromagnetic energy of large-scale Alfvén waves is not significant for the energy budget of highly accelerated field-aligned ions streaming at the lobeward edge of PSBL (i.e. relaxation length of these plasma flows is much larger than the distance from their source to the Earth). We also did not find the unique dependence of the kinetic energy flux upon the values of the corresponding AL index (not shown). It seems that it is necessary to perform the more accurate analysis of the local (in MLT) geomagnetic activity to make reliable conclusions. This will be a subject of a future publication.

\section{Conclusion}

On the basis of the analysis of 170 PSBL crossings made by Cluster spacecraft in the Earth's magnetotail (at $X=-13$ to $-19 R_{\mathrm{E}}$ ) we obtained the conclusive experimental evidence that sudden increases of drift velocities of low-energy lobe ion population, which are often observed at the PSBL-lobe interface, are caused by the ultra-low frequency wavy perturbations of the PSBL magnetic flux tubes generated due to the propagation of high velocity field-aligned ions. These fluc- tuations propagate earthward approximately with the local Alfvén velocity. We have statistically shown that the typical periods of the PSBL Alfvén fluctuations associated with high velocity ions are $\sim 1-4$ min and the typical wavelengths are $\sim 5-20 R_{\mathrm{E}}$. We have demonstrated that in the majority of cases from our data set high velocity field-aligned ions streaming earthward in the PSBL represented the ion component of high velocity flows. This finding allowed us to consider the Kelvin-Helmholtz instability as the most probable candidate for the generation of the observed magnetic fluctuations in frequency range $0.004-0.02 \mathrm{~Hz}$.

The investigation of the K-H instability was performed in a bounded flow sandwiched between the CPS and the lobe. Although this model is simplified relative to the real physical system, the obtained results are in a reasonable agreement with the observations. It was found that a development of the K-H instability leads to the formation of kink-like ultra-low frequency oscillations, resembling the experimentally observed ones. The wavelengths of the most unstable oscillations lie in approximately the same range as the ones experimentally measured.

Values of the Poynting flux carried by the PSBL Alfvén waves and estimated at $X=-13$ to $-19 R_{\mathrm{E}}$ in the majority of our cases do not exceed $0.01 \times 10^{-3} \mathrm{~J} / \mathrm{m}^{2} \mathrm{~s}$. However, the number of such weak events decreases with the increase of $|\mathrm{AL}|$ index and during active intervals (when $\mathrm{AL}<-600 \mathrm{nT}$ ) the Poynting flux registered in the PSBL may reach $0.08 \times 10^{-3} \mathrm{~J} / \mathrm{m}^{2}$ s, i.e. $0.08 \mathrm{~J} / \mathrm{m}^{2} \mathrm{~s}$, when mapped to the altitude $\sim 100 \mathrm{~km}$. However, even such large values of the electromagnetic energy flux form only few percents of the kinetic energy flux transferred by high velocity field-aligned ions streaming at the lobeward edge of PSBL. This implies that the losses of ion net energy for the excitation of the ultralow frequency unstable modes are very small and should have little influence on the characteristics of high velocity plasma flows.

Acknowledgements. The authors thank the Cluster CIS, PEACE, FGM and EFW experiment teams for providing the data. This work was supported by RFBR grants Nr. 10-02-00135; 10-0293114, 10-02-93115 and grant of Leading Scientific Schools HIII3200.2010.2.

Topical Editor I. A. Daglis thanks W. Lennartsson and A. Lui for their help in evaluating this paper.

\section{References}

Andrews, M. K., Daly, P. W., and Keppler, E.: Ion jetting at the plasma sheet boundary layer: Simultaneous observations of incident and reflected particles, Geophys. Res. Lett., 8, 987-990, 1981.

Angelopoulos, V., Elphic, R. C., Gary, S. P., and Huang, C. Y.: Electromagnetic instabilities in the plasma sheet boundary layer, J. Geophys. Res., 94(A11), 15373-15383, 1989.

Angelopoulos, V., Chapman, J. A., Mozer, F. S., Scudder, J. D., Russell C. T., Tsuruda, K., Mukai, T., Hughes, T. J., and Yumoto, 
K.: Plasma sheet electromagnetic power generation and its dissipation along auroral field lines, J. Geophys. Res., 107(A8), 1181, doi:10.1029/2001JA900136, 2002.

Baumjohann, W., Paschmann, G., Sckopke, N., Cattell, C. A., and Carlson C. W.: Average ion moments in the plasma sheet boundary layer, J. Geophys. Res., 93, 11507-11520, 1988.

Balogh, A., Carr, C. M., Acuña, M. H., Dunlop, M. W., Beek, T. J., Brown, P., Fornacon, K.-H., Georgescu, E., Glassmeier, K.H., Harris, J., Musmann, G., Oddy, T., and Schwingenschuh, K.: The Cluster Magnetic Field Investigation: overview of in-flight performance and initial results, Ann. Geophys., 19, 1207-1217, 2001, http://www.ann-geophys.net/19/1207/2001/.

Blumen, W.: Shear layer instability of an inviscid compressible fluid, J. Fluid Mech., 40, 769-779, 1970.

Burinskaya, T. M.: Kelvin-Helmholtz instability in a bounded flow, Plasma Physics Rep., 34(N11), 1013-1019, 2008.

Burinskaya, T. M., Shevelev, M. M., and Rauch, J.-L.: KelvinHelmholtz instability for a bounded plasma flow in the longitudinal magnetic field, Plasma Physics Rep., accepted, 2010.

Chanteur, G.: Spatial interpolation for four spacecraft: Theory in Analysis Methods for Multi-Spacecraft Data, edited by: Paschmann, G. and Daly, P. W., 349-360, European Space Agency, Paris, 1998.

Chaston, C. C., Bonnell, J. W., Carlson, C. W., McFadden, J. P., and Ergun, R. E.: Auroral ion acceleration in dispersive Alfvén waves, J. Geophys. Res., 109, A04205, doi:10.1029/2003JA010053, 2004.

DeCoster, R. J. and Frank, L. A.: Observations pertaining to the dynamics of the plasma sheet, J. Geophys. Res., 84, 5099-5121, 1979.

Eastman, T. E., Frank, L. A., Peterson, W. K., and Lennartsson, W.: The plasma sheet boundary layer, J. Geophys. Res., 89, 15531572, 1984.

Gary, S. P. and Winske, D.: Computer simulations of electromagnetic instabilities in the plasma sheet boundary layer, J. Geophys. Res., 95(A6), 8085-8094, 1990.

Grigorenko, E. E., Fedorov, A., and Zelenyi, L. M.: Statistical study of transient plasma structures in magnetotail lobes and plasma sheet boundary layer: Interball-1 observations, Ann. Geophys., 20, 329-340, 2002,

http://www.ann-geophys.net/20/329/2002/.

Grigorenko, E. E., Sauvaud, J.-A., and Zelenyi, L. M.: SpatialTemporal characteristics of ion beamlets in the plasma sheet boundary layer of magnetotail, J. Geophys. Res., 112, A05218, doi:10.1029/2006JA011986, 2007.

Grigorenko, E. E., Hoshino, M., Hirai, M., Mukai, T., and Zelenyi, L. M.: "Geography" of ion acceleration in the magnetotail. Xline versus Current Sheet effects, J. Geophys. Res., 114, A03203, doi:10.1029/2008JA013811, 2009.

Gustafsson, G., André, M., Carozzi, T., Eriksson, A. I., Fälthammar, C.-G., Grard, R., Holmgren, G., Holtet, J. A., Ivchenko, N., Karlsson, T., Khotyaintsev, Y., Klimov, S., Laakso, H., Lindqvist, P.-A., Lybekk, B., Marklund, G., Mozer, F., Mursula, K., Pedersen, A., Popielawska, B., Savin, S., Stasiewicz, K., Tanskanen, P., Vaivads, A., and Wahlund, J.-E.: First results of electric field and density observations by Cluster EFW based on initial months of operation, Ann. Geophys., 19, 1219-1240, 2001 , http://www.ann-geophys.net/19/1219/2001/.

Keiling, A., Wygant, J. R., Cattell, C., Temerin, M., Mozer, F. S., Kletzing, C. A., Scudder, J., Russell, S. T., Lotko, W., and Streltsov, A. V.: Large Alfvén wave power in the plasma sheet boundary layer during the expansion phase of substorms, Geophys. Res. Lett., 27(19), 3169-3172, 2000.

Keiling, A., Wygant, J. R., Cattell, C., Johnson, M., Temerin, M., Mozer, F. S., Kletzing, C. A., Scudder, J., and Russell, C. T.: Properties of large electric fields in the plasma sheet at 4-7 $R_{\mathrm{E}}$ measured with Polar, J. Geophys. Res., 106(A4), 5779-5798, 2001.

Keiling, A., Parks, G. K., Wygant, J. R., Dombeck, J., Mozer, F. S., Russell, C. T., Streltsov, A. V., and Lotko, W.: Some properties of Alfvén waves: observations in the tail lobes and the plasma sheet boundary layer, J. Geophys. Res., 110, A10S11, doi:10.1029/2004JA010907, 2005.

Keiling, A., Parks, G. K., Rème, H., Dandouras, I., Wilber, M., Kistler, L., Owen, C., Fazakerley, A. N., Lucek, E., Maksimovic, M., and Cornilleau-Wehrlin, N.: Energy-dispersed ions in the plasma sheet boundary layer and associated phenomena: Ion heating, electron acceleration, Alfvn waves, broadband waves, perpendicular electric field spikes, and auroral emissions, Ann. Geophys., 24, 2685-2707, 2006,

http://www.ann-geophys.net/24/2685/2006/.

Keiling, A.: Alfvén waves and their roles in the dynamics of the Earth's magnetotail: a review, Space Sci. Rev., 142, 73-156, 2009.

Lui, A. T. Y., Hones, E. W., Yasuhara, Jr. F., Akasofu, S.-I., and Bame S. J.: Magnetotail plasma flow during plasma sheet expansions: VELA 5 and 6 and IMP 6 observations, J. Geophys. Res., 82, 1235-1244, 1977.

Miura, A. and Pritchett, P. L.: Nonlocal stability analysis of the MHD Kelvin-Helmholtz instability in a compressible plasma, J. Geophys. Res., 87, 7431-7444, 1982.

Mukai, T., Hirahara, M., Machida, S., Saito, Y., Terasawa, T., Nishida A.: Geotail observations of cold ion streams in the medium distance magnetotail lobe in the course of a substorm, Geophys. Res. Lett., 21(11), 1023-1026, 1994.

Owen, C. J., Fazakerley, A. N., Carter, P. J., Coates, A. J., Krauklis, I. C., Szita, S., Taylor, M. G. G. T., Travnicek, P., Watson, G., Wilson, R. J., Balogh, A., and Dunlop, M. W.: Cluster PEACE observations of electrons during magnetospheric flux transfer events, Ann. Geophys., 19, 1509-1522, 2001, http://www.ann-geophys.net/19/1509/2001/.

Parks, G., Chen, L. J., McCarthy, M., Larson, D., Lin, R. P., Phan, T., Reme, H., and Sanderson, T.: New observations of ion beams in the plasma sheet boundary layer, Geophys. Res. Lett., 25(17), 3285-3288, 1998.

Rème, H., Aoustin, C., Bosqued, J. M., Dandouras, I., Lavraud, B., Sauvaud, J. A., Barthe, A., Bouyssou, J., Camus, Th., CoeurJoly, O., Cros, A., Cuvilo, J., Ducay, F., Garbarowitz, Y., Medale, J. L., Penou, E., Perrier, H., Romefort, D., Rouzaud, J., Vallat, C., Alcaydé, D., Jacquey, C., Mazelle, C., d’Uston, C., Möbius, E., Kistler, L. M., Crocker, K., Granoff, M., Mouikis, C., Popecki, M., Vosbury, M., Klecker, B., Hovestadt, D., Kucharek, H., Kuenneth, E., Paschmann, G., Scholer, M., Sckopke, N., Seidenschwang, E., Carlson, C. W., Curtis, D. W., Ingraham, C., Lin, R. P., McFadden, J. P., Parks, G. K., Phan, T., Formisano, V., Amata, E., Bavassano-Cattaneo, M. B., Baldetti, P., Bruno, R., Chion- 
chio, G., Di Lellis, A., Marcucci, M. F., Pallocchia, G., Korth, A., Daly, P. W., Graeve, B., Rosenbauer, H., Vasyliunas, V., McCarthy, M., Wilber, M., Eliasson, L., Lundin, R., Olsen, S., Shelley, E. G., Fuselier, S., Ghielmetti, A. G., Lennartsson, W., Escoubet, C. P., Balsiger, H., Friedel, R., Cao, J.-B., Kovrazhkin, R. A., Papamastorakis, I., Pellat, R., Scudder, J., and Sonnerup, B.: First multispacecraft ion measurements in and near the Earth's magnetosphere with the identical Cluster ion spectrometry (CIS) experiment, Ann. Geophys., 19, 1303-1354, 2001, http://www.ann-geophys.net/19/1303/2001/.

Sauvaud, J.-A., Louarn, P., Fruit, G., Stenuit, H., Vallat, C., Dandouras, J., Rème, H., André, M., Balogh, A., Dunlop, M., Kistler, L., Möbius, E., Mouikis, C., Klecker, B., Parks, G. K., McFadden, J., Carlson, C., Marcucci, F., Pallocchia, G., Lundin, R., Korth, A., and McCarthy, M.: Case studies of the dynamics of ionospheric ions in the Earth's magnetotail., J. Geophys. Res., 109, A01212, doi:10.1029/2003JA009996, 2004.

Sharp, R. D., Carr, D. L., Peterson, W. K., and Shelley, E. G.: Ion streams in the magnetotail, J. Geophys. Res., 86, 4639-4648, 1981.

Takahashi, K. and Hones, E. W.: ISEE 1 and 2 observations of ion distributions at the plasma sheet - tail lobe boundary, J. Geophys. Res., 93, 8558-8582, 1988.
Takada, T., Seki, K., Hirahara, M., Fujimoto, M., Saito, Y., Hayakawa, H., and Mukai T.: Statistical properties of lowfrequency waves and ion beams in the plasma sheet boundary layer: Geotail observations, J. Geophys. Res., 110, A02204, doi:10.1029/2004JA010395, 2005.

Williams, D. J.: Energetic ion beams at the edge of the plasma sheet: ISSE 1 observations plus a simple explanatory model, J. Geophys. Res., 86, 5501-5518, 1981.

Wygant, J. R., Keiling, A., Cattell, C. A., Johnson, M., Lysak, R. L., Temerin, M., Mozer, F. S., Kletzing, C. A., Scudder, J., Peterson, W., Russell, S. T., Parks, G., Brittnacher, M., Germany, G., and Spann, J.: Polar spacecraft based comparisons of intense electric fields and the Poynting flux near and within the plasma sheet tail lobe boundary to UVI images: An energy source to the aurora, J. Geophys. Res., 105, 18675-18692, 2000.

Zelenyi, L. M., Grigorenko, E. E., and Fedorov, A. O.: Spatialtemporal ion structures in the Earth's magnetotail: beamlets as a result of nonadiabatic impulse acceleration of the plasma, JETP Letters, 80(10), 663-673, 2004. 